\title{
Banach spaces of bounded Szlenk index
}

\author{
by \\ E. Odell (Austin, TX), Th. Schlumprecht (College Station, TX) \\ and A. ZsÁK (Nottingham)
}

\begin{abstract}
For a countable ordinal $\alpha$ we denote by $\mathcal{C}_{\alpha}$ the class of separable, reflexive Banach spaces whose Szlenk index and the Szlenk index of their dual are bounded by $\alpha$. We show that each $\mathcal{C}_{\alpha}$ admits a separable, reflexive universal space. We also show that spaces in the class $\mathcal{C}_{\omega^{\alpha} \cdot \omega}$ embed into spaces of the same class with a basis. As a consequence we deduce that each $\mathcal{C}_{\alpha}$ is analytic in the Effros-Borel structure of subspaces of $C[0,1]$.
\end{abstract}

1. Introduction. A well known result that dates back to the early days of Banach space theory [4, Théorème 9 , p. 185] states that every separable Banach space embeds into $C[0,1]$, i.e., that $C[0,1]$ is universal for the class of all separable Banach spaces. Pełczyński [23] refined this result by showing that there are Banach spaces $X$ with a basis and $X_{\mathrm{u}}$ with an unconditional basis such that every space with a basis or with an unconditional basis is isomorphic to a complemented subspace of $X$ or $X_{\mathrm{u}}$, respectively.

By a famous result of Szlenk [26], there is no separable reflexive Banach space $X$ which contains isomorphically all separable reflexive Banach spaces. Bourgain [7] sharpened this result by showing that a separable Banach space which contains all separable reflexive Banach spaces must contain $C[0,1]$ and, thus, all separable Banach spaces. An isometric relative of Bourgain's result has recently been shown by Kalton and Godefroy [13]: if a separable Banach space contains an isometric copy of every separable strictly convex Banach space, then it is isometrically universal. Szlenk proved his result by introducing for a Banach space $X$ an ordinal index $\mathrm{Sz}(X)$ (see Section 5 below) which is countable if and only if $X$ has a separable dual and is hereditary (if $Y$ embeds in $X$, then $\mathrm{Sz}(Y) \leq \mathrm{Sz}(X)$ ). Moreover he showed that for any countable ordinal $\alpha$ there is a separable reflexive space $X$ for which $\operatorname{Sz}(X)>\alpha$. Bourgain achieved his result by introducing an index

2000 Mathematics Subject Classification: 46B20, 54H05.

Key words and phrases: Szlenk index, universal space, embedding into FDDs, EffrosBorel structure, analytic classes.

Research of the first two authors was supported by the National Science Foundation. 
which measures how well finite sections of the Schauder basis of $C[0,1]$ embed in $X$, and then he proved statements analogous to Szlenk's approach.

Bourgain then raised the question whether there is a separable, reflexive space that contains isomorphically all uniformly convex Banach spaces. This problem was solved recently by the first two authors. It was proven in [20] that if $X$ is a separable, uniformly convex Banach space, then there exist $1<q \leq p<\infty$ and a reflexive space $Z$ with an FDD (finite-dimensional decomposition $\left(E_{n}\right)$ so that $X$ embeds into $Z$ and $\left(E_{n}\right)$ satisfies block $\left(\ell_{p}, \ell_{q}\right)$ estimates. This means that for some $C$ we have

$$
C^{-1}\left(\sum\left\|z_{i}\right\|^{p}\right)^{1 / p} \leq\left\|\sum z_{i}\right\| \leq C\left(\sum\left\|z_{i}\right\|^{q}\right)^{1 / q}
$$

for any block sequence $\left(z_{i}\right)$ of $\left(E_{n}\right)$. In fact this holds (see [21, Theorems 3.4 and 4.1]) if we merely know that

$$
X \in \mathcal{C}_{\omega}=\left\{Y: Y \text { is separable, reflexive, } \mathrm{Sz}(Y) \leq \omega, \mathrm{Sz}\left(Y^{*}\right) \leq \omega\right\} .
$$

Using then a result of S. Prus [24], who solved Bourgain's question within the class of Banach spaces with FDDs, we deduce that there exists a universal reflexive space $Z$ for the class $\mathcal{C}_{\omega}$, and in fact $Z \in \mathcal{C}_{\omega^{2}}$.

Inspired by the results of [20], A. Pełczyński raised the question whether a similar result could be proved for the classes $\mathcal{C}_{\alpha}$, where $\alpha<\omega_{1}$ and where $\mathcal{C}_{\alpha}$ is defined analogously to the class $\mathcal{C}_{\omega}$. This would mean that, although the class of separable, reflexive spaces has no universal element, it is the (necessarily uncountable) increasing union of classes which are closed under taking duals, and for which universal separable, reflexive spaces do exist. In this paper we answer this in the affirmative.

As in the proof in [20], we will reduce the universality problem to an embedding problem. We will show that any member of $\mathcal{C}_{\alpha}, \alpha<\omega_{1}$, embeds into some element of $\mathcal{C}_{\beta}$ which has a basis, where $\alpha \leq \beta<\omega_{1}$ depends on $\alpha$. This result can be seen as a quantitative version of Zippin's seminal theorem [28] that every separable reflexive space embeds into one with a basis. In light of our embedding result we then only need to show that the class of elements in $\mathcal{C}_{\alpha}$ with a basis admits a universal separable, reflexive space. Let us mention here a different quantitative version of Zippin's theorem by Bossard [5]: for every $\alpha<\omega_{1}$ there exists $\beta<\omega_{1}$ such that every separable space with Szlenk index at most $\alpha$ embeds into a Banach space with shrinking basis and with Szlenk index at most $\beta$. Our embedding result is very different, since the Szlenk index of a separable space does not control the Szlenk index of its dual.

Our approach depends on showing that if $X$ is a space with separable dual and if $\mathrm{Sz}(X) \leq \omega^{\alpha \cdot \omega}$ for some $\alpha<\omega_{1}$, then $X$ satisfies "subsequential upper $T_{\alpha, c}$ estimates", where $T_{\alpha, c}$ is the Tsirelson space defined by the Schreier class $\mathcal{S}_{\alpha}$ and a parameter $c \in(0,1)$ (the definitions of $\mathcal{S}_{\alpha}$ and $T_{\alpha, c}$ will be recalled 
in Section 3). Subsequential upper $T_{\alpha, c}$ estimates can be expressed in terms of a game played as follows. Player (I) starts by choosing $X_{1} \in \operatorname{cof}(X)$, the set of all finite-codimensional subspaces of $X$, and an integer $k_{1} \in \mathbb{N}$. Player (II) then responds by selecting $x_{1} \in S_{X_{1}}$, the unit sphere of $X_{1}$. Then (I) chooses $X_{2} \in \operatorname{cof}(X)$ and $k_{2} \in \mathbb{N}$, and (II) chooses $x_{2} \in S_{X_{2}}$, etc. The space $X$ satisfies subsequential upper $T_{\alpha, c}$ estimates if for some $C<\infty$ Player (I) has a winning strategy to force (II) to select $\left(x_{i}\right)$ satisfying

$$
\left\|\sum a_{i} x_{i}\right\|_{X} \leq C\left\|\sum a_{i} t_{k_{i}}\right\|_{T_{\alpha, c}}
$$

for all $\left(a_{i}\right) \subset \mathbb{R}$, where $\left(t_{i}\right)$ is the unit vector basis of $T_{\alpha, c}$. These games are a variation of those introduced in [19] and were defined and analysed in [22]. Using the results therein we ultimately prove the following structure theorem.

TheOREm A. Let $\alpha<\omega_{1}$. For a separable, reflexive space $X$ the following are equivalent:

(i) $X \in \mathcal{C}_{\omega^{\alpha \cdot \omega}}$.

(ii) $X$ embeds into a separable, reflexive space $Z$ with an FDD $\left(E_{i}\right)$ which satisfies subsequential $\left(T_{\alpha, c}^{*}, T_{\alpha, c}\right)$ estimates in $Z$ for some $c \in(0,1)$.

In part (ii) "subsequential $\left(T_{\alpha, c}^{*}, T_{\alpha, c}\right)$ estimates" mean the following: there exists $C<\infty$ such that if $\left(z_{i}\right)$ is a block sequence of $\left(E_{n}\right)$ with min $\operatorname{supp}\left(z_{i}\right)$ $=k_{i}$, then

$$
C^{-1}\left\|\sum\right\| z_{i}\left\|t_{k_{i}}^{*}\right\|_{T_{\alpha, c}^{*}} \leq\left\|\sum z_{i}\right\| \leq C\left\|\sum\right\| z_{i}\left\|t_{k_{i}}\right\|_{T_{\alpha, c}} .
$$

Of course the implication "(ii) $\Rightarrow$ (i)" shows that the space $Z$ lies in the same class $\mathcal{C}_{\omega^{\alpha \cdot \omega}}$ as $X$ does.

Roughly speaking, the Tsirelson spaces $T_{\alpha, c}$ of order $\alpha$ form a sort of upper envelope and their duals $T_{\alpha, c}^{*}$ form a lower envelope for the entire class $\mathcal{C}_{\omega^{\alpha \cdot \omega}}$. Moreover, since the spaces $T_{\alpha, c}$ also belong to the class $\mathcal{C}_{\omega^{\alpha \cdot \omega}}$, this result is best possible.

From Theorem A and the main result of [22] (see Theorem 1 below) we will then deduce the following embedding and universality result.

TheOREM B. For each $\alpha<\omega_{1}$ every Banach space in the class $\mathcal{C}_{\omega^{\alpha} \cdot \omega}$ embeds into a space $Z$ with a basis that lies in the same class $\mathcal{C}_{\omega^{\alpha \cdot \omega}}$ as $X$ does.

THEOREM C. For each $\alpha<\omega_{1}$ there is an element of $\mathcal{C}_{\omega^{\alpha \cdot \omega+1}}$ with a basis which is universal for the class $\mathcal{C}_{\omega^{\alpha \cdot \omega}}$.

Our structural result, Theorem A, will be proved in Section 6 together with some more general structural results (Theorem 21, Corollary 20). We will then deduce FDD versions (see Theorem 23) of our embedding result, 
Theorem B, and our universality result, Theorem C. A result of W. B. Johnson [14] allows us to replace FDDs by bases in our conclusions (see Theorem 24 and the proof of Theorems B and C thereafter). In Sections 2 to 5 we present the relevant notation and background material: the embedding theorem from [22], Tsirelson spaces, general ordinal indices and the Szlenk index. Each of these sections begins with a brief summary of its contents.

There is a completely different approach to universality problems that uses tools of descriptive set theory. There have been some remarkable achievements in Banach space theory using such techniques, including solutions of universality problems $[2,11]$. However, in order to tackle Pełczyński's question with this approach, one would need the classes $\mathcal{C}_{\alpha}$ to be analytic and this was not known. Note that if we knew the function $\max \left\{\operatorname{Sz}(X), \mathrm{Sz}\left(X^{*}\right)\right\}$ to be a co-analytic rank on the class of separable, reflexive spaces, then we could deduce that the classes $\mathcal{C}_{\alpha}$ are in fact Borel. Whether $\max \left\{\operatorname{Sz}(X), \operatorname{Sz}\left(X^{*}\right)\right\}$ is a co-analytic rank is still an open problem: it is mentioned by Bossard in [6], where he shows that this function is a co-analytic rank on the class of bases of reflexive spaces. Our results, however, now do show the following.

TheOREM D. For each countable ordinal $\alpha$ the class $\mathcal{C}_{\alpha}$ is analytic in the Effros-Borel structure of closed subspaces of $C[0,1]$.

If $\alpha$ is of the form $\omega^{\eta \cdot \omega}$ for some $\eta<\omega_{1}$, then Theorem $\mathrm{D}$ follows from our main results and from standard facts in descriptive set theory (as pointed out to us by C. Rosendal). This, combined with a recent result of P. Dodos [10] concerning analyticity of duals of analytic classes, then gives the general case. We present this result in the final section of our paper. We are grateful to P. Dodos, V. Ferenczi and C. Rosendal for showing us the descriptive-settheoretic implications of our results.

Let us now mention some open problems. The first one asks if Theorem $\mathrm{C}$ can be sharpened.

Problem. Is there a universal element of $\mathcal{C}_{\omega^{\alpha \cdot \omega}}$ for each $\alpha<\omega_{1}$ ?

We do know that there is no space $Z$ in $\mathcal{C}_{\omega^{\alpha} \cdot \omega}$ such that for some $K>0$ every space in $\mathcal{C}_{\omega^{\alpha \cdot \omega}} K$-embeds into $Z$ (see the Remark following Theorem 22). We also know that the answer to the above question is negative if the Tsirelson spaces $T_{\alpha, c}$ are of bounded distortion with constant $D$ independent of $c \in[1 / 2,1)$. Of course, it is a famous, long standing open problem whether even the Tsirelson space $T_{1,1 / 2}$ is of bounded distortion.

It is known that one only needs to consider classes $\mathcal{C}_{\alpha}$ where $\alpha$ is of the form $\omega^{\eta}$ for some $\eta<\omega_{1}$ (Theorem 12 in Section 5). In Section 6 we obtain embedding and universality results for these general classes (Theorem 22). However, these are not quite as sharp as Theorems B and C above. This leads to the following questions. 
Problem. Is it true that, given $\alpha<\omega_{1}$, every space in $\mathcal{C}_{\omega^{\alpha}}$ embeds into a space with a basis of the same class?

Is there a universal element of $\mathcal{C}_{\omega^{\alpha}}$ for each $\alpha<\omega_{1}$ ?

2. Embeddings into spaces with FDDs. In this section we state an embedding theorem from [22] (Theorem 1 below). This requires a fair amount of definitions. Much of this will be used throughout the paper.

Let $Z$ be a Banach space with an FDD $E=\left(E_{n}\right)$. For $n \in \mathbb{N}$ we denote by $P_{n}^{E}$ the $n$th coordinate projection, i.e., $P_{n}^{E}: Z \rightarrow E_{n}$ is the map defined by $\sum_{i} z_{i} \mapsto z_{n}$, where $z_{i} \in E_{i}$ for all $i \in \mathbb{N}$. For a finite set $A \subset \mathbb{N}$ we put $P_{A}^{E}=\sum_{n \in A} P_{n}^{E}$. The projection constant $K(E, Z)$ of $\left(E_{n}\right)($ in $Z)$ is defined by

$$
K=K(E, Z)=\sup _{m \leq n}\left\|P_{[m, n]}^{E}\right\|
$$

where $[m, n]$ denotes the interval $\{m, m+1, \ldots, n\}$ in $\mathbb{N}$. Recall that $K$ is always finite and, as in the case of bases, we say that $\left(E_{n}\right)$ is bimonotone (in $Z$ ) if $K=1$. By passing to the equivalent norm

$$
\|\cdot\|: Z \rightarrow \mathbb{R}, \quad z \mapsto \sup _{m \leq n}\left\|P_{[m, n]}^{E}(z)\right\|,
$$

we can always renorm $Z$ so that $K=1$.

A sequence $\left(F_{n}\right)$ of finite-dimensional spaces is called a blocking of $\left(E_{n}\right)$ if for some sequence $m_{1}<m_{2}<\cdots$ in $\mathbb{N}$ we have $F_{n}=\bigoplus_{j=m_{n-1}+1}^{m_{n}} E_{j}$ for all $n \in \mathbb{N}\left(m_{0}=0\right)$. Note that if $E=\left(E_{n}\right)$ is an FDD of a Banach space $Z$, and if $F=\left(F_{n}\right)$ is a blocking of $\left(E_{n}\right)$, then $\left(F_{n}\right)$ is also an FDD for $Z$ with $K(F, Z) \leq K(E, Z)$.

For a sequence $\left(E_{i}\right)$ of finite-dimensional spaces we define the vector space $\mathrm{c}_{00}\left(\bigoplus_{i=1}^{\infty} E_{i}\right)=\left\{\left(z_{i}\right): z_{i} \in E_{i}\right.$ for all $i \in \mathbb{N}$, and $\left\{i \in \mathbb{N}: z_{i} \neq 0\right\}$ is finite $\}$

which is dense in each Banach space for which $\left(E_{i}\right)$ is an FDD. For a set $A \subset \mathbb{N}$ we denote by $\bigoplus_{i \in A} E_{i}$ the linear subspace of $c_{00}\left(\bigoplus E_{i}\right)$ generated by the elements of $\bigcup_{i \in A} E_{i}$. As usual we denote by $c_{00}$ the vector space of sequences in $\mathbb{R}$ which are eventually zero. We will sometimes consider for the same sequence $\left(E_{i}\right)$ of finite-dimensional spaces different norms on $\mathrm{c}_{00}\left(\bigoplus E_{i}\right)$. In order to avoid confusion we will therefore often index the norm by the Banach space whose norm we are using, i.e., $\|\cdot\|_{Z}$ denotes the norm of the Banach space $Z$.

If $Z$ has an $\operatorname{FDD}\left(E_{i}\right)$, then the vector space $c_{00}\left(\bigoplus_{i=1}^{\infty} E_{i}^{*}\right)$, where $E_{i}^{*}$ is the dual space of $E_{i}$ for each $i \in \mathbb{N}$, can be identified in a natural way with a $w^{*}$-dense subspace of $Z^{*}$. Note however that the embedding $E_{i}^{*} \hookrightarrow Z^{*}$ is, in general, not isometric unless $K=1$. We will always consider $E_{i}^{*}$ with the 
norm it inherits from $Z^{*}$ instead of the norm it has as the dual space of $E_{i}$. We denote the norm closure of $c_{00}\left(\bigoplus_{i=1}^{\infty} E_{i}^{*}\right)$ in $Z^{*}$ by $Z^{(*)}$. Note that $Z^{(*)}$ is $w^{*}$-dense in $Z^{*}$, the unit ball $B_{Z^{(*)}}$ norms $Z$, and $\left(E_{i}^{*}\right)$ is an $\operatorname{FDD}$ of $Z^{(*)}$ having a projection constant not exceeding $K(E, Z)$. If $K(E, Z)=1$, then $B_{Z^{(*)}}$ is 1-norming for $Z$ and $Z^{(*)(*)}=Z$.

For $z \in \mathrm{c}_{00}\left(\bigoplus E_{i}\right)$ we define the support $\operatorname{supp}_{E}(z)$ of $z$ with respect to $\left(E_{i}\right)$ by

$$
\operatorname{supp}_{E}(z)=\left\{i \in \mathbb{N}: P_{i}^{E}(z) \neq 0\right\}
$$

A sequence $\left(z_{i}\right)$ (finite or infinite) of non-zero vectors in $c_{00}\left(\bigoplus E_{i}\right)$ is called a block sequence of $\left(E_{i}\right)$ if

$$
\max \operatorname{supp}_{E}\left(z_{n}\right)<\min \operatorname{supp}_{E}\left(z_{n+1}\right) \quad \text { for } n \in \mathbb{N}\left(\text { or } n<\operatorname{length}\left(z_{i}\right)\right) \text {. }
$$

A block sequence $\left(z_{i}\right)$ of $\left(E_{i}\right)$ is called normalized (in $Z$ ) if $\left\|z_{n}\right\|_{Z}=1$ for all $n$.

Let $\bar{\delta}=\left(\delta_{i}\right) \subset(0,1)$ with $\delta_{i} \downarrow 0$. A (finite or infinite) sequence $\left(z_{i}\right)$ in $S_{Z}$ is called a $\bar{\delta}$-block sequence of $\left(E_{i}\right)$ if there exists a sequence $0 \leq k_{0}<k_{1}<$ $k_{2}<\cdots$ in $\mathbb{N}$ such that

$$
\left.\left\|z_{n}-P_{\left(k_{n-1}, k_{n}\right]}^{E}\left(z_{n}\right)\right\|<\delta_{n} \quad \text { for all } n \in \mathbb{N} \text { (or } n \leq \operatorname{length}\left(z_{i}\right)\right) .
$$

Definition. Given two sequences $\left(e_{i}\right)$ and $\left(f_{i}\right)$ in some Banach spaces, and given a constant $C>0$, we say that $\left(f_{i}\right) C$-dominates $\left(e_{i}\right)$, or that $\left(e_{i}\right)$ is $C$-dominated by $\left(f_{i}\right)$, if

$$
\left\|\sum a_{i} e_{i}\right\| \leq C\left\|\sum a_{i} f_{i}\right\| \quad \text { for all }\left(a_{i}\right) \in \mathrm{c}_{00} .
$$

We say that $\left(e_{i}\right)$ and $\left(f_{i}\right)$ are $C$-equivalent if there exist positive constants $A$ and $B$ with $A \cdot B \leq C$ such that $\left(f_{i}\right) A$-dominates $\left(e_{i}\right)$ and is $B$-dominated by $\left(e_{i}\right)$.

We say that $\left(f_{i}\right)$ dominates $\left(e_{i}\right)$, or that $\left(e_{i}\right)$ is dominated by $\left(f_{i}\right)$, if there exists a constant $C>0$ such that $\left(f_{i}\right) C$-dominates $\left(e_{i}\right)$. We say that $\left(e_{i}\right)$ and $\left(f_{i}\right)$ are equivalent if they are $C$-equivalent for some $C>0$.

We shall now introduce certain lower and upper norm estimates for FDDs.

Definition. Let $Z$ be a Banach space with an FDD $\left(E_{n}\right)$, let $V$ be a Banach space with a normalized, 1 -unconditional basis $\left(v_{i}\right)$ and let $1 \leq C<\infty$.

We say that $\left(E_{n}\right)$ satisfies subsequential $C$-V-lower estimates (in $Z$ ) if every normalized block sequence $\left(z_{i}\right)$ of $\left(E_{n}\right)$ in $Z C$-dominates $\left(v_{m_{i}}\right)$, where $m_{i}=\operatorname{minsupp}_{E}\left(z_{i}\right)$ for all $i \in \mathbb{N}$, and $\left(E_{n}\right)$ satisfies subsequential $C-V$ upper estimates (in $Z$ ) if every normalized block sequence $\left(z_{i}\right)$ of $\left(E_{n}\right)$ in $Z$ is $C$-dominated by $\left(v_{m_{i}}\right)$, where $m_{i}=\min \operatorname{supp}_{E}\left(z_{i}\right)$ for all $i \in \mathbb{N}$. 
If $U$ is another space with a normalized and 1 -unconditional basis $\left(u_{i}\right)$, we say that $\left(E_{n}\right)$ satisfies subsequential $C-(V, U)$ estimates $($ in $Z)$ if it satisfies subsequential $C$ - $V$-lower and $C$ - $U$-upper estimates in $Z$.

We say that $\left(E_{n}\right)$ satisfies subsequential $V$-lower, $U$-upper or $(V, U)$ estimates (in $Z$ ) if for some $C \geq 1$ it satisfies subsequential $C$ - $V$-lower, $C$ - $U$ upper or $C$ - $(V, U)$ estimates in $Z$, respectively.

We shall need a coordinate-free version of subsequential lower and upper estimates. This can be done in terms of a game as described in the Introduction. Another way uses infinite, countably branching trees, and this is what we shall follow here. For $l \in \mathbb{N}$ we define

$$
T_{l}=\left\{\left(n_{1}, \ldots, n_{l}\right): n_{1}<\cdots<n_{l} \text { are in } \mathbb{N}\right\}
$$

and

$$
T_{\infty}=\bigcup_{l=1}^{\infty} T_{l}, \quad T_{\infty}^{\text {even }}=\bigcup_{l=1}^{\infty} T_{2 l} .
$$

An even tree in a Banach space $X$ is a family $\left(x_{\alpha}\right)_{\alpha \in T_{\infty}^{\text {even }}}$ in $X$. Sequences of the form $\left(x_{(\alpha, n)}\right)_{n>n_{2 l-1}}$, where $l \in \mathbb{N}$ and $\alpha=\left(n_{1}, \ldots, n_{2 l-1}\right) \in T_{\infty}$, are called nodes of the tree. For a sequence $n_{1}<n_{2}<\cdots$ of positive integers the sequence $\left(x_{\left(n_{1}, \ldots, n_{2 l}\right)}\right)_{l=1}^{\infty}$ is called a branch of the tree.

An even tree $\left(x_{\alpha}\right)_{\alpha \in T_{\infty}^{\text {even }}}$ in a Banach space $X$ is called normalized if $\left\|x_{\alpha}\right\|=1$ for all $\alpha \in T_{\infty}^{\text {even }}$, and weakly null if every node is a weakly null

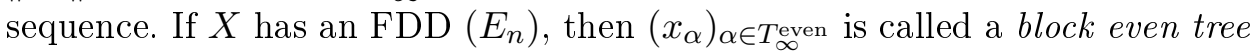
of $\left(E_{n}\right)$ if every node is a block sequence of $\left(E_{n}\right)$.

Definition. Let $V$ be a Banach space with a normalized and 1-unconditional basis $\left(v_{i}\right)$, and let $C \in[1, \infty)$. Let $X$ be an infinite-dimensional Banach space. We say that $X$ satisfies subsequential $C$-V-lower tree estimates if every normalized, weakly null even tree $\left(x_{\alpha}\right)_{\alpha \in T_{\infty}^{\text {even }}}$ in $X$ has a branch $\left(x_{\left(n_{1}, \ldots, n_{2 i}\right)}\right)$ which $C$-dominates $\left(v_{n_{2 i-1}}\right)$.

We say that $X$ satisfies subsequential $C$ - $V$-upper tree estimates if every normalized, weakly null even tree $\left(x_{\alpha}\right)_{\alpha \in T_{\infty}^{\text {even }}}$ in $X$ has a branch $\left(x_{\left(n_{1}, \ldots, n_{2 i}\right)}\right)$ which is $C$-dominated by $\left(v_{n_{2 i-1}}\right)$.

If $U$ is a second space with a 1 -unconditional and normalized basis $\left(u_{i}\right)$, we say that $X$ satisfies subsequential $C-(V, U)$ tree estimates if it satisfies subsequential $C$ - $V$-lower and $C$ - $U$-upper tree estimates.

We say that $X$ satisfies subsequential $V$-lower, $U$-upper or $(V, U)$ tree estimates if, for some $1 \leq C<\infty, X$ satisfies subsequential $C$ - $V$-lower, $C$ - $U$-upper or $C$ - $(V, U)$ tree estimates, respectively.

We next define some properties of bases which appear in the statement of Theorem 1. 
Definition. Let $V$ be a Banach space with a normalized, 1-unconditional basis $\left(v_{i}\right)$ and let $1 \leq C<\infty$. We say that $\left(v_{i}\right)$ is $C$-block-stable if any two normalized block bases $\left(x_{i}\right)$ and $\left(y_{i}\right)$ with

$$
\max \left(\operatorname{supp}\left(x_{i}\right) \cup \operatorname{supp}\left(y_{i}\right)\right)<\min \left(\operatorname{supp}\left(x_{i+1}\right) \cup \operatorname{supp}\left(y_{i+1}\right)\right) \quad \text { for all } i \in \mathbb{N}
$$

are $C$-equivalent. We say that $\left(v_{i}\right)$ is block-stable if it is $C$-block-stable for some constant $C$.

We say that $\left(v_{i}\right)$ is $C$-right-dominant (respectively, $C$-left-dominant) if for all sequences $m_{1}<m_{2}<\cdots$ and $n_{1}<n_{2}<\cdots$ of positive integers with $m_{i} \leq n_{i}$ for all $i \in \mathbb{N},\left(v_{m_{i}}\right)$ is $C$-dominated by (respectively, $C$-dominates) $\left(v_{n_{i}}\right)$. We say that $\left(v_{i}\right)$ is right-dominant or left-dominant if for some $C \geq 1$ it is $C$-right-dominant or $C$-left-dominant, respectively.

We are now ready to state the main embedding theorem from [22] which we shall use in the proofs of the main results of this paper.

Let $V$ and $U$ be reflexive spaces with normalized, 1-unconditional, blockstable bases $\left(v_{i}\right)$ and $\left(u_{i}\right)$, respectively, such that $\left(v_{i}\right)$ is left-dominant, $\left(u_{i}\right)$ is right-dominant and $\left(v_{i}\right)$ is dominated by $\left(u_{i}\right)$. For each $C \in[1, \infty)$ let $\mathcal{A}_{V, U}(C)$ denote the class of all separable, infinite-dimensional, reflexive Banach spaces that satisfy subsequential $C-(V, U)$-tree estimates. We also let

$$
\mathcal{A}_{V, U}=\bigcup_{C \in[1, \infty)} \mathcal{A}_{V, U}(C),
$$

which is the class of all separable, infinite-dimensional, reflexive Banach spaces that satisfy subsequential $(V, U)$-tree estimates.

ThEOREM $1([22])$. The class $\mathcal{A}_{V, U}$ contains a universal element. More precisely, for all $B, D, L, R \in[1, \infty)$ there exists a constant $\bar{C}=\bar{C}(B, D) \in$ $[1, \infty)$ and for all $C \in[1, \infty)$ there is a constant $K(C)=K_{B, D, L, R}(C) \in$ $[1, \infty)$ such that if $\left(v_{i}\right)$ is B-block-stable and L-left-dominant, if $\left(u_{i}\right)$ is $B$ block-stable and $R$-right-dominant, and if $\left(v_{i}\right)$ is D-dominated by $\left(u_{i}\right)$, then there exists $Z \in \mathcal{A}_{V, U}$ such that every $X \in \mathcal{A}_{V, U}(C) K(C)$-embeds into $Z$, and moreover $Z$ has a bimonotone FDD satisfying subsequential $\bar{C}-(V, U)$ estimates in $Z$.

At some point we shall also need the following duality result.

Proposition 2 ([22]). Assume that $U$ is a space with a normalized, 1-unconditional basis $\left(u_{i}\right)$ which is $R$-right-dominant for some $R \geq 1$, and that $X$ is a reflexive space which satisfies subsequential $C$-U-upper tree estimates for some $C \geq 1$. Then, for any $\varepsilon>0, X^{*}$ satisfies subsequential $(2 C R+\varepsilon)-U^{(*)}$-lower tree estimates.

3. Tsirelson spaces. When we apply Theorem 1 we shall take $U=T_{\alpha}$, the Tsirelson space of order $\alpha$ with parameter $1 / 2$, and $V=T_{\alpha}^{*}$, the dual 
of $T_{\alpha}$. In this section we recall the definition and some of the properties of $T_{\alpha}$. At the end we will state a combinatorial principle which will be used later on.

We begin with some preliminary definitions. We shall write $[\mathbb{N}]^{<\omega}$ for the set of all finite subsets of $\mathbb{N}$, and $[\mathbb{N}]^{\omega}$ for the set of all infinite subsets of $\mathbb{N}$. These two families will be given the product topology as subsets of $\{0,1\}^{\mathbb{N}}$. A family $\mathcal{F} \subset[\mathbb{N}]^{<\omega}$ is called hereditary if $A \in \mathcal{F}$ whenever $A \subset B$ and $B \in \mathcal{F}$, and $\mathcal{F}$ is called compact if it is compact in the product topology. Note that a hereditary family is compact if and only if it contains no strictly ascending chains. A family $\mathcal{F} \subset[\mathbb{N}]^{<\omega}$ is called thin if, for all $A, B \in \mathcal{F}$, from $A \subset B$ it follows that $A=B$, i.e., $\mathcal{F}$ contains no two comparable (with respect to inclusion) elements.

Given $n, a_{1}<\cdots<a_{n}, b_{1}<\cdots<b_{n}$ in $\mathbb{N}$ we say that $\left\{b_{1}, \ldots, b_{n}\right\}$ is a spread of $\left\{a_{1}, \ldots, a_{n}\right\}$ if $a_{i} \leq b_{i}$ for $i=1, \ldots, n$. A family $\mathcal{F} \subset[\mathbb{N}]^{<\omega}$ is called spreading if every spread of every element of $\mathcal{F}$ is also in $\mathcal{F}$. This is an appropriate place to make the convention that the elements of a subset of $\mathbb{N}$ will always be written in increasing order. So, for example, when we write $\left\{m_{1}, \ldots, m_{k}\right\} \in[\mathbb{N}]^{<\omega}$, it is implicitly assumed that $m_{1}<\cdots<m_{k}$.

For $\mathcal{F} \subset[\mathbb{N}]^{<\omega}$ we write $\operatorname{MAX}(\mathcal{F})$ for the set of maximal (with respect to inclusion) elements of $\mathcal{F}$. Note that $\operatorname{MAX}(\mathcal{F})$ is always a thin family.

For subsets $A$ and $B$ of $\mathbb{N}$ we write $A<B$ if $a<b$ for all $a \in A$ and $b \in B$. For $n \in \mathbb{N}$ and $A \subset \mathbb{N}$ we write $n<A$ if $\{n\}<A$. A (finite or infinite) sequence $A_{1}, A_{2}, \ldots$ of subsets of $\mathbb{N}$ is called successive if $A_{1}<A_{2}<\cdots$. Given a family $\mathcal{F} \subset[\mathbb{N}]^{<\omega}$, a sequence $A_{1}, \ldots, A_{k}$ of non-empty, finite subsets of $\mathbb{N}$ is called $\mathcal{F}$-admissible if it is successive and $\left\{\min A_{1}, \ldots, \min A_{k}\right\} \in \mathcal{F}$.

We next recall the definitions of the Schreier families $\mathcal{S}_{\alpha}$ and the fine Schreier families $\mathcal{F}_{\alpha}$, where $\alpha$ is a countable ordinal. We first fix for every limit ordinal $\lambda$ a sequence $\left(\alpha_{n}\right)$ of ordinals with $1 \leq \alpha_{n} \nearrow \lambda$. We now define the fine Schreier families $\left(\mathcal{F}_{\alpha}\right)_{\alpha<\omega_{1}}$ by recursion:

$$
\begin{aligned}
\mathcal{F}_{0} & =\{\emptyset\}, \\
\mathcal{F}_{\alpha+1} & =\left\{\{n\} \cup A: n \in \mathbb{N}, A \in \mathcal{F}_{\alpha}, n<A\right\} \cup\{\emptyset\}, \\
\mathcal{F}_{\lambda} & =\left\{A \in[\mathbb{N}]^{<\omega}: \exists n \leq \min A, A \in \mathcal{F}_{\alpha_{n}}\right\},
\end{aligned}
$$

where in the last line $\lambda$ is a limit ordinal and $\alpha_{n} \nearrow \lambda$ is the sequence of ordinals fixed in advance. An easy induction shows that $\mathcal{F}_{\alpha}$ is a compact, hereditary and spreading family for all $\alpha<\omega_{1}$. Moreover, $\left(\mathcal{F}_{\alpha}\right)_{\alpha<\omega_{1}}$ is an "almost" increasing chain:

$$
\forall \alpha \leq \beta<\omega_{1} \exists n \in \mathbb{N} \forall F \in \mathcal{F}_{\alpha}, \quad \text { if } n \leq \min F \text {, then } F \in \mathcal{F}_{\beta} .
$$

This can be proved by an easy induction on $\beta$. We also note that for $A \in$ $\mathcal{F}_{\alpha} \backslash \operatorname{MAX}\left(\mathcal{F}_{\alpha}\right)$ we have $A \cup\{n\} \in \mathcal{F}_{\alpha}$ for all $n>\max A$. 
The Schreier families can now be defined by setting $\mathcal{S}_{\alpha}=\mathcal{F}_{\omega^{\alpha}}$ for all $\alpha<\omega_{1}$. This is not exactly how the Schreier families are usually defined, but it gives the same families provided we are more careful when choosing the sequences $\left(\alpha_{n}\right)$ with $1 \leq \alpha_{n} \nearrow \lambda$ for limit ordinals $\lambda$ (these choices should depend on the choices made when defining the Schreier families in the usual way). At any rate, what matters is that each $\mathcal{S}_{\alpha}$ be a compact, hereditary and spreading family with Cantor-Bendixson index $\omega^{\alpha}+1$ (see (2) in Section 4). Note that $\mathcal{S}_{1}$ is the usual Schreier family $\mathcal{S}$ given by

$$
\mathcal{S}=\left\{A \in[\mathbb{N}]^{<\omega}:|A| \leq \min A\right\}
$$

(provided that for $\lambda=\omega$ we chose the sequence $\alpha_{n}=n$ ).

As usual we denote by $\left(e_{i}\right)$ the canonical (algebraic) basis of the vector space $\mathrm{c}_{00}$ of all eventually zero scalar sequences. For $x=\sum x_{i} e_{i} \in \mathrm{c}_{00}$ and for $A \subset \mathbb{N}$ we write $A x$ for the obvious projection of $x$ onto $\operatorname{span}\left\{e_{i}: i \in A\right\}$ :

$$
A x=\sum_{i \in A} x_{i} e_{i} .
$$

We are now ready to recall the definitions of certain Tsirelson type spaces. For a compact, hereditary family $\mathcal{F} \subset[\mathbb{N}]^{<\omega}$ and for $c \in(0,1)$ there is a unique least norm on $c_{00}$, denoted by $\|\cdot\|_{\mathcal{F}, c}$, such that $\|x\|_{\mathcal{F}, c}=\|x\|_{\infty} \vee c \cdot \sup \left\{\sum_{i=1}^{n}\left\|A_{i} x\right\|_{\mathcal{F}, c}: n \in \mathbb{N}, A_{1}, \ldots, A_{n}\right.$ is $\mathcal{F}$-admissible $\}$ for all $x \in \mathrm{c}_{00}$. We shall write $T_{\mathcal{F}, c}$ for the completion of $\mathrm{c}_{00}$ in this norm. Note that the (algebraic) basis $\left(e_{i}\right)$ of $\mathrm{c}_{00}$ becomes a 1-unconditional (Schauder) basis of $T_{\mathcal{F}, c}$.

For a non-zero, countable ordinal $\alpha$ and for $c \in(0,1)$ the space $T_{\mathcal{S}_{\alpha}, c}$ is the Tsirelson space of order $\alpha$ with parameter $c$; we shall denote it by $T_{\alpha, c}$. We further simplify notation in the case $c=1 / 2$ by letting $T_{\alpha}=T_{\alpha, 1 / 2}$. When $\alpha=1$ this is just (the dual of) the original Tsirelson space $[27,12]$.

We gather some properties of Tsirelson spaces in the next proposition. In particular, we note that the unit vector bases of $T_{\alpha}$ and $T_{\alpha}^{*}$ satisfy the conditions required in Theorem 1.

Proposition 3 ([8], [18]). Let $\alpha$ be a non-zero, countable ordinal. The Tsirelson space $T_{\alpha}$ is a reflexive Banach space and $\left(e_{i}\right)$ is a 1-unconditional, 1-right-dominant and B-block-stable basis for $T_{\alpha}$, where $B$ is a constant independent of $\alpha$.

The biorthogonal functionals $\left(e_{i}^{*}\right)$ form a 1-unconditional, 1-left dominant and B-block-stable basis for $T_{\alpha}^{*}$. Moreover, $\left(e_{i}^{*}\right)$ is D-dominated by $\left(e_{i}\right)$, where $D$ is a universal constant.

It is shown in [8] that Tsirelson's space $T_{1}$ is block-stable (see also [9, Proposition II.4]). The argument easily carries over to higher order Tsirelson 
spaces giving the same constant. A proof is given in [18] for an even larger class of Tsirelson type spaces.

It is proved in $\left[9\right.$, Proposition V.10] that the unit vector basis $\left(e_{i}\right)$ of Tsirelson's space $T_{1}$ dominates the unit vector basis of $\ell_{q}$ for all $q>1$. The last statement of Proposition 3 now follows immediately. (Note that $\mathcal{S}_{1} \subset \mathcal{S}_{\alpha}$, and hence the unit vector basis of $T_{1}$ is 1-dominated by the unit vector basis of $T_{\alpha}$ for any $1 \leq \alpha<\omega_{1}$.)

The rest of the properties claimed in Proposition 3 are immediate from the definition of the higher order Tsirelson spaces.

We end this section by stating a combinatorial theorem of Pudlák and Rödl which also follows from infinite Ramsey theory. This has nothing to do with Tsirelson spaces, but as it concerns families of finite subsets of $\mathbb{N}$, this section is an appropriate place for it.

THEOREM 4. Let $\mathcal{F} \subset[\mathbb{N}]^{<\omega}$ be a thin family. Whenever each element of $\mathcal{F}$ is coloured red or blue, there is an infinite subset $M$ of $\mathbb{N}$ such that $\mathcal{F} \cap[M]^{<\omega}$ is monochromatic, where $[M]^{<\omega}$ denotes the set of all finite subsets of $M$.

4. Ordinal indices. The main aim of this section is to introduce two ordinal indices in Banach spaces: the weak index and the block index. The former will be related to the Szlenk index later on. In order to avoid tiresome repetitions we begin by defining a class of ordinal indices of trees on arbitrary sets. We then introduce the said indices as special cases and prove a number of their properties to be used in what follows.

Let $X$ be an arbitrary set. We define $X^{<\omega}=\bigcup_{n=0}^{\infty} X^{n}$, the set of all finite sequences in $X$, which includes the sequence of length zero, denoted by $\emptyset$. For $x \in X$ we shall write $x$ instead of $(x)$, i.e., we identify $X$ with sequences of length 1 in $X$. A tree on $X$ is a non-empty subset $\mathcal{F}$ of $X^{<\omega}$ closed under taking initial segments: if $\left(x_{1}, \ldots, x_{n}\right) \in \mathcal{F}$ and $0 \leq m \leq n$, then $\left(x_{1}, \ldots, x_{m}\right) \in \mathcal{F}$. A tree $\mathcal{F}$ on $X$ is hereditary if every subsequence of every member of $\mathcal{F}$ is also in $\mathcal{F}$.

Given $\mathbf{x}=\left(x_{1}, \ldots, x_{m}\right)$ and $\mathbf{y}=\left(y_{1}, \ldots, y_{n}\right)$ in $X^{<\omega}$, we write $(\mathbf{x}, \mathbf{y})$ for the concatenation of $\mathbf{x}$ and $\mathbf{y}$ :

$$
(\mathbf{x}, \mathbf{y})=\left(x_{1}, \ldots, x_{m}, y_{1}, \ldots, y_{n}\right) .
$$

Given $\mathcal{F} \subset X^{<\omega}$ and $\mathbf{x} \in X^{<\omega}$, we let

$$
\mathcal{F}(\mathbf{x})=\left\{\mathbf{y} \in X^{<\omega}:(\mathbf{x}, \mathbf{y}) \in \mathcal{F}\right\} .
$$

Note that if $\mathcal{F}$ is a tree on $X$, then so is $\mathcal{F}(\mathbf{x})$ (unless it is empty). Moreover, if $\mathcal{F}$ is hereditary, then so is $\mathcal{F}(\mathbf{x})$ and $\mathcal{F}(\mathbf{x}) \subset \mathcal{F}$.

Let $X^{\omega}$ denote the set of all (infinite) sequences in $X$. Fix $S \subset X^{\omega}$. For a tree $\mathcal{F}$ on $X$ the $S$-derivative $\mathcal{F}_{S}^{\prime}$ of $\mathcal{F}$ consists of all finite sequences 
$\mathbf{x} \in X^{<\omega}$ for which there is a sequence $\left(y_{i}\right)_{i=1}^{\infty} \in S$ with $\left(\mathbf{x}, y_{i}\right) \in \mathcal{F}$ for all $i \in \mathbb{N}$. Note that $\mathcal{F}_{S}^{\prime} \subset \mathcal{F}$ and that $\mathcal{F}_{S}^{\prime}$ is also a tree (unless it is empty). We then define higher order derivatives $\mathcal{F}_{S}^{(\alpha)}$ for ordinals $\alpha<\omega_{1}$ by recursion as follows:

$$
\begin{array}{rlrl}
\mathcal{F}_{S}^{(0)} & =\mathcal{F}, & \\
\mathcal{F}_{S}^{(\alpha+1)} & =\left(\mathcal{F}_{S}^{(\alpha)}\right)_{S}^{\prime} & & \text { for all } \alpha<\omega_{1}, \\
\mathcal{F}_{S}^{(\lambda)} & =\bigcap_{\alpha<\lambda} \mathcal{F}_{S}^{(\alpha)} & & \text { for a limit ordinal } \lambda<\omega_{1} .
\end{array}
$$

It is clear that $\mathcal{F}_{S}^{(\alpha)} \supset \mathcal{F}_{S}^{(\beta)}$ whenever $\alpha \leq \beta$ and that $\mathcal{F}_{S}^{(\alpha)}$ is a tree (or the empty set) for all $\alpha$. An easy induction also shows that

$$
(\mathcal{F}(\mathbf{x}))_{S}^{(\alpha)}=\left(\mathcal{F}_{S}^{(\alpha)}\right)(\mathbf{x}) \quad \text { for all } \mathbf{x} \in X^{<\omega}, \alpha<\omega_{1} .
$$

We now define the $S$-index $\mathrm{I}_{S}(\mathcal{F})$ of $\mathcal{F}$ by

$$
\mathrm{I}_{S}(\mathcal{F})=\min \left\{\alpha<\omega_{1}: \mathcal{F}_{S}^{(\alpha)}=\emptyset\right\}
$$

if there exists $\alpha<\omega_{1}$ with $\mathcal{F}_{S}^{(\alpha)}=\emptyset$, and $\mathrm{I}_{S}(\mathcal{F})=\omega_{1}$ otherwise.

REMARK. If $\lambda$ is a limit ordinal and $\mathcal{F}_{S}^{(\alpha)} \neq \emptyset$ for all $\alpha<\lambda$, then in particular $\emptyset \in \mathcal{F}_{S}^{(\alpha)}$ for all $\alpha<\lambda$, and hence $\mathcal{F}_{S}^{(\lambda)} \neq \emptyset$. This shows that $\mathrm{I}_{S}(\mathcal{F})$ is always a successor ordinal.

EXAMPLES. 1. A hereditary family $\mathcal{F} \subset[\mathbb{N}]^{<\omega}$ can be thought of as a tree on $\mathbb{N}$ : a set $F=\left\{m_{1}, \ldots, m_{k}\right\} \in[\mathbb{N}]^{<\omega}$ is identified with $\left(m_{1}, \ldots, m_{k}\right) \in \mathbb{N}<\omega$ (recall that $m_{1}<\cdots<m_{k}$ by our convention of always listing the elements of a subset of $\mathbb{N}$ in increasing order).

Let $S$ be the set of all strictly increasing sequences in $\mathbb{N}$. In this case the $S$-index of a compact, hereditary family $\mathcal{F} \subset[\mathbb{N}]^{<\omega}$ is nothing else than the Cantor-Bendixson index of $\mathcal{F}$ as a compact topological space, which we will denote by $\mathrm{I}_{\mathrm{CB}}(\mathcal{F})$. We will also use the term "Cantor-Bendixson derivative" instead of " $S$-derivative" and use the notation $\mathcal{F}_{\mathrm{CB}}^{\prime}$ and $\mathcal{F}_{\mathrm{CB}}^{(\alpha)}$.

2. If $X$ is an arbitrary set and $S=X^{\omega}$, then the $S$-index of a tree $\mathcal{F}$ on $X$ is what is usually called the order (or height) of $\mathcal{F}$, denoted by o $(\mathcal{F})$. Note that in this case the $S$-derivative of $\mathcal{F}$ consists of all finite sequences $\mathbf{x} \in X^{<\omega}$ for which there exists $y \in X$ such that $(\mathbf{x}, y) \in \mathcal{F}$.

The function $\mathrm{o}(\cdot)$ is the largest index: for any $S \subset X^{\omega}$ we have $\mathrm{o}(\mathcal{F}) \geq \mathrm{I}_{S}(\mathcal{F})$.

We say that $S \subset X^{\omega}$ contains diagonals if every subsequence of every member of $S$ also belongs to $S$ and for every sequence $\left(\mathbf{x}_{n}\right)$ in $S$ with $\mathbf{x}_{n}=$ $\left(x_{n, i}\right)_{i=1}^{\infty}$ there exist $i_{1}<i_{2}<\cdots$ in $\mathbb{N}$ such that $\left(x_{n, i_{n}}\right)_{n=1}^{\infty}$ belongs to $S$. 
If $S$ contains diagonals, then the $S$-index of a tree on $X$ may be measured via the Cantor-Bendixson index of the fine Schreier families $\left(\mathcal{F}_{\alpha}\right)_{\alpha<\omega_{1}}$ introduced earlier. An easy induction argument shows that

$$
\mathrm{I}_{\mathrm{CB}}\left(\mathcal{F}_{\alpha}\right)=\alpha+1 \quad \text { for all } \alpha<\omega_{1} .
$$

Given a tree $\mathcal{F} \subset[\mathbb{N}]^{<\omega}$ on $\mathbb{N}$, a family $\left(x_{F}\right)_{F \in \mathcal{F} \backslash\{\emptyset\}}$ in $X$ will always be viewed as the tree

$$
\left\{\left(x_{\left\{m_{1}\right\}}, x_{\left\{m_{1}, m_{2}\right\}}, \ldots, x_{\left\{m_{1}, m_{2}, \ldots, m_{k}\right\}}\right): k \geq 0,\left\{m_{1}, \ldots, m_{k}\right\} \in \mathcal{F}\right\}
$$

on $X$.

Proposition 5. Let $X$ be an arbitrary set and let $S \subset X^{\omega}$. If $S$ contains diagonals, then for a tree $\mathcal{F}$ on $X$ and for a countable ordinal $\alpha$ the following are equivalent:

(i) $\alpha<\mathrm{I}_{S}(\mathcal{F})$.

(ii) There is a family $\left(x_{F}\right)_{F \in \mathcal{F}_{\alpha} \backslash\{\emptyset\}} \subset \mathcal{F}$ such that for all $F \in \mathcal{F}_{\alpha} \backslash$ $\operatorname{MAX}\left(\mathcal{F}_{\alpha}\right)$ the sequence $\left(x_{F \cup\{n\}}\right)_{n>\max F}$ is in $S$.

Proof. "(ii) $\Rightarrow$ (i)" An easy induction on $\beta<\omega_{1}$ shows that for all $F=$ $\left\{m_{1}, \ldots, m_{k}\right\} \in\left(\mathcal{F}_{\alpha}\right)_{\mathrm{CB}}^{(\beta)}$ we have

$$
\left(x_{\left\{m_{1}\right\}}, x_{\left\{m_{1}, m_{2}\right\}}, \ldots, x_{\left\{m_{1}, m_{2}, \ldots, m_{k}\right\}}\right) \in \mathcal{F}_{S}^{(\beta)} .
$$

It follows that $\mathrm{I}_{S}(\mathcal{F}) \geq \mathrm{I}_{\mathrm{CB}}\left(\mathcal{F}_{\alpha}\right)>\alpha$.

"(i) $\Rightarrow$ (ii)" We prove this by induction on $\alpha$. When $\alpha=0$, statement (ii) says that $\emptyset \in \mathcal{F}$, which does follow from $0<\mathrm{I}_{S}(\mathcal{F})$.

Next assume that $\alpha+1<\mathrm{I}_{S}(\mathcal{F})$. Then $\mathcal{F}_{S}^{(\alpha+1)} \neq \emptyset$, so in particular we have $\emptyset \in \mathcal{F}_{S}^{(\alpha+1)}$. It follows that there is a sequence $\left(x_{i}\right)_{i=1}^{\infty} \in S$ such that $x_{i} \in \mathcal{F}_{S}^{(\alpha)}$ for all $i \in \mathbb{N}$. Hence $\left(\mathcal{F}_{S}^{(\alpha)}\right)\left(x_{i}\right)=\left(\mathcal{F}\left(x_{i}\right)\right)_{S}^{(\alpha)}$ is non-empty, and $\mathrm{I}_{S}\left(\mathcal{F}\left(x_{i}\right)\right)>\alpha$. By the induction hypothesis, for each $i \in \mathbb{N}$ there is a family $\left(y_{i, F}\right)_{F \in \mathcal{F}_{\alpha} \backslash\{\emptyset\}} \subset \mathcal{F}\left(x_{i}\right)$ such that for all $F \in \mathcal{F}_{\alpha} \backslash \operatorname{MAX}\left(\mathcal{F}_{\alpha}\right)$ the sequence $\left(y_{i, F \cup\{n\}}\right)_{n>\max F}$ is in $S$.

Now for each $F=\left\{m_{1}, \ldots, m_{k}\right\} \in \mathcal{F}_{\alpha+1}$ define

$$
x_{F}= \begin{cases}x_{i} & \text { if } k=1 \text { and } m_{1}=i, \\ y_{i,\left\{m_{2}, m_{3}, \ldots, m_{k}\right\}} & \text { if } k>1 \text { and } m_{1}=i .\end{cases}
$$

It is routine to verify that statement (ii) holds with $\alpha+1$ replacing $\alpha$.

Finally, let $\lambda$ be a limit ordinal, and assume that $\lambda<\mathrm{I}_{S}(\mathcal{F})$. Let $\left(\alpha_{n}\right)$ be the sequence of ordinals with $1 \leq \alpha_{n} \nearrow \lambda$ chosen in the definition of the fine Schreier family $\mathcal{F}_{\lambda}$. By the induction hypothesis, for each $n \in \mathbb{N}$ there is a family $\left(y_{n, F}\right)_{F \in \mathcal{F}_{\alpha_{n}} \backslash\{\emptyset\}} \subset \mathcal{F}$ such that for all $F \in \mathcal{F}_{\alpha_{n}} \backslash \operatorname{MAX}\left(\mathcal{F}_{\alpha_{n}}\right)$ the sequence $\left(y_{n, F \cup\{i\}}\right)_{i>\max F}$ is in $S$. In particular we have $\left(y_{n,\{i\}}\right)_{i=1}^{\infty} \in S$ for all $n \in \mathbb{N}$. Since $S$ contains diagonals there exist $i_{1}<i_{2}<\cdots$ in $\mathbb{N}$ such 
that $\left(y_{n,\left\{i_{n}\right\}}\right)_{n=1}^{\infty} \in S$. We can also ensure that if $m \leq n, F \in \mathcal{F}_{\alpha_{m}}$ and $i_{n} \leq \min F$, then $F \in \mathcal{F}_{\alpha_{n}}$ (see (1)).

Now for each $F=\left\{m_{1}, \ldots, m_{k}\right\} \in \mathcal{F}_{\lambda}$ define

$$
x_{F}= \begin{cases}y_{n,\left\{i_{n}\right\}} & \text { if } k=1 \text { and } m_{1}=n, \\ y_{n,\left\{i_{n}, i_{n}+m_{2}, i_{n}+m_{3}, \ldots, i_{n}+m_{k}\right\}} & \text { if } k>1 \text { and } m_{1}=n .\end{cases}
$$

It is again routine to verify that statement (ii) with $\lambda$ in place of $\alpha$ follows.

The set $S=X^{\omega}$ for an arbitrary set $X$, and the set $S$ used to define the Cantor-Bendixson index for a compact, hereditary family in $[\mathbb{N}]^{<\omega}$, trivially contain diagonals. This will also be (mostly) the case in the following two examples of $S$-indices in Banach spaces.

EXAmples. 1. The weak index. Let $X$ be a separable Banach space. Let $S$ be the set of all weakly null sequences in $S_{X}$, the unit sphere of $X$. We call the $S$-index of a tree $\mathcal{F}$ on $S_{X}$ the weak index of $\mathcal{F}$ and we denote it by $\mathrm{I}_{\mathrm{w}}(\mathcal{F})$. We shall use the term "weak derivative" instead of " $S$-derivative" and use the notation $\mathcal{F}_{\mathrm{w}}^{\prime}$ and $\mathcal{F}_{\mathrm{w}}^{(\alpha)}$.

When the dual space $X^{*}$ is separable, the weak topology on the unit ball $B_{X}$ of $X$ is metrizable. Hence in this case the set $S$ contains diagonals and Proposition 5 applies.

2. The block index. Let $X$ be a Banach space with an FDD $E=\left(E_{i}\right)$. A block tree of $\left(E_{i}\right)$ in $Z$ is a tree $\mathcal{F}$ on $S_{X}$ such that every element of $\mathcal{F}$ is a (finite) block sequence of $\left(E_{i}\right)$. Let $S$ be the set of all normalized, infinite block sequences of $\left(E_{i}\right)$ in $Z$. We call the $S$-index of a block tree $\mathcal{F}$ of $\left(E_{i}\right)$ the block index of $\mathcal{F}$ and we denote it by $\mathrm{I}_{\mathrm{bl}}(\mathcal{F})$. We shall use the term "block derivative" instead of "S-derivative" and use the notation $\mathcal{F}_{\text {bl }}^{\prime}$ and $\mathcal{F}_{\text {bl }}^{(\alpha)}$. Note that the set $S$ contains diagonals, and hence Proposition 5 applies.

Note also that $\left(E_{i}\right)$ is a shrinking FDD of $X$ if and only if every element of $S$ is weakly null. In this case we have

$$
\mathrm{I}_{\mathrm{bl}}(\mathcal{F}) \leq \mathrm{I}_{\mathrm{w}}(\mathcal{F})
$$

for any block tree $\mathcal{F}$ of $\left(E_{i}\right)$ in $Z$. The converse is false in general, but it is true up to perturbations and without the assumption that $\left(E_{i}\right)$ is shrinking (see the Remark preceding Proposition 8 below).

REMARK. If $\left(E_{i}\right)$ and $\left(F_{i}\right)$ are two different FDDs of the Banach space $X$, then the corresponding block indices they give rise to may well be different in general. However, it is clear that if $\left(F_{i}\right)$ is a blocking of $\left(E_{i}\right)$, then they do yield the same block index. Since this is exactly the kind of situation in which we shall use the block index in this paper, we did not incorporate the underlying FDD in the notation for block derivatives and for the block index. 
In the next section we will relate the Szlenk index to the weak index of certain trees. In the rest of the present section we prove two propositions. The first one is a perturbation result: it concerns the weak index of the "fattening" of a tree. The second result relates the block index of block trees in Banach spaces to the Cantor-Bendixson index of compact, hereditary families in $[\mathbb{N}]^{<\omega}$. It is a kind of discretization result.

Let $X$ be a separable Banach space. For a tree $\mathcal{F} \subset S_{X}^{<\omega}$ and for $\bar{\varepsilon}=$ $\left(\varepsilon_{i}\right) \subset(0,1)$ we write

$\mathcal{F}_{\bar{\varepsilon}}^{X}=\left\{\left(x_{i}\right)_{i=1}^{n} \in S_{X}^{<\omega}: n \in \mathbb{N}, \exists\left(y_{i}\right)_{i=1}^{n} \in \mathcal{F},\left\|x_{i}-y_{i}\right\| \leq \varepsilon_{i}\right.$ for $\left.i=1, \ldots, n\right\}$.

Proposition 6. Let $X \subset Y$ be Banach spaces with separable duals, and let $\mathcal{F} \subset S_{X}^{<\omega}$ be a tree on $S_{X}$. Then for all $\bar{\varepsilon}=\left(\varepsilon_{i}\right) \subset(0,1)$ we have $\mathrm{I}_{\mathrm{w}}\left(\mathcal{F}_{\bar{\varepsilon}}^{Y}\right) \leq \mathrm{I}_{\mathrm{w}}\left(\mathcal{F}_{\overline{5} \bar{\varepsilon}}^{X}\right)$.

Proof. By a theorem of Zippin [28], $Y$ embeds into a Banach space with a shrinking FDD. So without loss of generality we may assume that $Y$ itself has a shrinking FDD $E=\left(E_{i}\right)$. Let $K=K(E, Y)$, the projection constant of $E$ in $Y$, and set

$$
X_{m}=X \cap \overline{\bigoplus_{j=m+1}^{\infty} E_{j}} \quad(m \in \mathbb{N}) .
$$

For $\varepsilon \in(0,1)$ and for $A \subset S_{Y}$ we define $A_{\varepsilon}^{Y}$ by

$$
A_{\varepsilon}^{Y}=\left\{y \in S_{Y}: \exists x \in A,\|x-y\| \leq \varepsilon\right\} .
$$

We shall need the following lemma.

Lemma 7. Let $\varepsilon \in(0,1)$ and let $\left(y_{i}\right)$ be a weakly null sequence in $\left(S_{X}\right)_{\varepsilon}^{Y}$. Then there is a weakly null sequence $\left(x_{i}\right)$ in $S_{X}$ and a subsequence $\left(y_{i}^{\prime}\right)$ of $\left(y_{i}\right)$ such that $\left\|x_{i}-y_{i}^{\prime}\right\| \leq 4 \varepsilon$ for all $i \in \mathbb{N}$.

Proof. Fix $\eta>0$ such that

$$
\varepsilon^{\prime}=(1+\eta)(2 \eta+\varepsilon)<1 \text { and } 2 \varepsilon^{\prime}+\varepsilon<4 \varepsilon .
$$

Let $m \in \mathbb{N}$. Since $\left(X / X_{m}\right)^{*} \cong X_{m}^{\perp}$ is a finite-dimensional subspace of $X^{*}$, there is a finite subset $A_{m}$ of $B_{X^{*}}$ such that

$$
d\left(x, X_{m}\right) \leq(1+\eta) \cdot \max _{f \in A_{m}} f(x) \quad \text { for all } x \in X .
$$

Let $B_{m}$ be a finite subset of $B_{Y^{*}}$ containing a Hahn-Banach extension to $Y$ of each element of $A_{m}$. Then choose $n(m, \eta) \in \mathbb{N}$ such that

$$
\left\|g-P_{[1, n(m, \eta)]}^{E^{*}}(g)\right\|<\eta \quad \text { for all } g \in B_{m} .
$$

Now let $\left(y_{i}^{\prime}\right)$ be a subsequence of $\left(y_{i}\right)$ such that

$$
\left\|P_{[1, n(m, \eta)]}^{E}\left(y_{m}^{\prime}\right)\right\|<\eta \quad \text { for all } m \in \mathbb{N} .
$$


For each $m \in \mathbb{N}$ choose $z_{m} \in S_{X}$ with $\left\|z_{m}-y_{m}^{\prime}\right\| \leq \varepsilon$. We have

$$
\begin{aligned}
d\left(z_{m}, X_{m}\right) & \leq(1+\eta) \cdot \max _{g \in B_{m}} g\left(z_{m}\right) \leq(1+\eta) \cdot\left(\max _{g \in B_{m}} g\left(y_{m}^{\prime}\right)+\varepsilon\right) \\
& <(1+\eta) \cdot\left(\max _{g \in B_{m}} P_{[1, n(m, \eta)]}^{E^{*}}(g)\left(y_{m}^{\prime}\right)+\eta+\varepsilon\right) \\
& \leq(1+\eta) \cdot\left(\left\|P_{[1, n(m, \eta)]}^{E}\left(y_{m}^{\prime}\right)\right\|+\eta+\varepsilon\right) \leq(1+\eta) \cdot(2 \eta+\varepsilon)=\varepsilon^{\prime} .
\end{aligned}
$$

Choose $\widetilde{x}_{m} \in X_{m}$ such that $\left\|\widetilde{x}_{m}-z_{m}\right\|<\varepsilon^{\prime}$, and set $x_{m}=\widetilde{x}_{m} /\left\|\widetilde{x}_{m}\right\|$. An easy computation shows that

$$
\left\|x_{m}-y_{m}^{\prime}\right\|<2 \varepsilon^{\prime}+\varepsilon<4 \varepsilon \quad \text { for all } m \in \mathbb{N} .
$$

Since $\left(E_{i}\right)$ is shrinking, it follows that the sequence $\left(x_{i}\right)$ is weakly null.

We now continue with the proof of Proposition 6. Let $\bar{\varepsilon}=\left(\varepsilon_{i}\right) \subset(0,1)$. It is enough to show that if $\alpha<\mathrm{I}_{\mathrm{w}}\left(\mathcal{F}_{\bar{\varepsilon}}^{Y}\right)$, then $\alpha<\mathrm{I}_{\mathrm{w}}\left(\mathcal{F}_{5 \bar{\varepsilon}}^{X}\right)$. Now if $\alpha<\mathrm{I}_{\mathrm{w}}\left(\mathcal{F}_{\bar{\varepsilon}}^{Y}\right)$, then by Proposition 5 there is a family $\left(y_{F}\right)_{F \in \mathcal{F}_{\alpha} \backslash\{\emptyset\}} \subset \mathcal{F}_{\bar{\varepsilon}}^{Y}$ such that for all $F \in \mathcal{F}_{\alpha} \backslash \operatorname{MAX}\left(\mathcal{F}_{\alpha}\right)$ the sequence $\left(y_{F \cup\{n\}}\right)_{n>\max F}$ is weakly null.

Given a spreading family $\mathcal{F} \subset[\mathbb{N}]^{<\omega}$ we will call a function $F \mapsto F^{\prime}$ : $\mathcal{F} \rightarrow \mathcal{F}$ a pruning function if for every $F=\left\{m_{1}, \ldots, m_{l}\right\} \in \mathcal{F}$ the set $F^{\prime}=\left\{m_{1}^{\prime}, \ldots, m_{l}^{\prime}\right\}$ is a spread of $F$ and $\left\{m_{1}, \ldots, m_{k}\right\}^{\prime}=\left\{m_{1}^{\prime}, \ldots, m_{k}^{\prime}\right\}$ for each $k=1, \ldots, l$. Now by repeated applications of Lemma 7 we can find a family $\left(x_{F}\right)_{F \in \mathcal{F}_{\alpha} \backslash\{\emptyset\}} \subset S_{X}$ and a pruning function $F \mapsto F^{\prime}: \mathcal{F}_{\alpha} \rightarrow \mathcal{F}_{\alpha}$ such that

$$
\left(x_{F \cup\{n\}}\right)_{n>\max F} \text { is weakly null for all } F \in \mathcal{F}_{\alpha} \backslash \operatorname{MAX}\left(\mathcal{F}_{\alpha}\right)
$$

and

$\left\|x_{F}-y_{F^{\prime}}\right\| \leq 4 \varepsilon_{i} \quad$ for all $i \in \mathbb{N}$ and for all $F \in \mathcal{F}_{\alpha}$ with $|F|=i$.

The last line implies that $\left(x_{F}\right)_{F \in \mathcal{F}_{\alpha} \backslash\{\emptyset\}} \subset \mathcal{F}_{5 \bar{\varepsilon}}^{X}$, and hence by Proposition 5 we have $\alpha<\mathrm{I}_{\mathrm{w}}\left(\mathcal{F}_{\overline{5}}^{X}\right)$, as required.

Let $Z$ be a Banach space with an FDD $E=\left(E_{i}\right)$, and let $\mathcal{F}$ be a block tree of $\left(E_{i}\right)$ in $Z$. Let us write $\Sigma(E, Z)$ for the set of all finite, normalized block sequences on $\left(E_{i}\right)$ in $Z$. For $\bar{\varepsilon}=\left(\varepsilon_{i}\right) \subset(0,1)$ we let

$$
\mathcal{F}_{\bar{\varepsilon}}^{E, Z}=\mathcal{F}_{\bar{\varepsilon}}^{Z} \cap \Sigma(E, Z),
$$

i.e., $\mathcal{F}_{\bar{\varepsilon}}^{E, Z}$ is the restriction to block sequences of the $\bar{\varepsilon}$-"fattening" of $\mathcal{F}$ in $Z$ :

$$
\begin{aligned}
\mathcal{F}_{\bar{\varepsilon}}^{E, Z}=\left\{\left(x_{i}\right)_{i=1}^{n} \in \Sigma(E, Z): n \in \mathbb{N}, \exists\left(y_{i}\right)_{i=1}^{n} \in \mathcal{F},\right. & \left\|x_{i}-y_{i}\right\| \leq \varepsilon_{i} \\
& \text { for } i=1, \ldots, n\} .
\end{aligned}
$$

We also define the compression $\widetilde{\mathcal{F}}$ of $\mathcal{F}$ by

$$
\widetilde{\mathcal{F}}=\left\{F \in[\mathbb{N}]^{<\omega}: \exists\left(z_{i}\right)_{i=1}^{|F|} \in \mathcal{F}, F=\left\{\min _{\operatorname{supp}}\left(z_{i}\right): i=1, \ldots,|F|\right\}\right\} .
$$

REMARK. Having introduced the above notation, we can now write down a sort of converse for (3). If $Z$ is a Banach space with an FDD $E=\left(E_{i}\right)$ and 
$\mathcal{F}$ is a block tree of $\left(E_{i}\right)$ in $Z$, then

$$
\mathrm{I}_{\mathrm{w}}(\mathcal{F}) \leq \mathrm{I}_{\mathrm{bl}}\left(\mathcal{F}_{\bar{\varepsilon}}^{E, Z}\right)
$$

for all $\bar{\varepsilon}=\left(\varepsilon_{i}\right) \subset(0,1)$. Indeed, if $\alpha<\mathrm{I}_{\mathrm{w}}(\mathcal{F})$, then by Proposition 5 there is a family $\left(x_{F}\right)_{F \in \mathcal{F}_{\alpha} \backslash\{\emptyset\}} \subset \mathcal{F}$ such that for all $F \in \mathcal{F}_{\alpha} \backslash \operatorname{MAX}\left(\mathcal{F}_{\alpha}\right)$ the sequence $\left(x_{F \cup\{n\}}\right)_{n>\max F}$ is weakly null. By standard perturbation arguments we get a pruning function $F \mapsto F^{\prime}: \mathcal{F}_{\alpha} \rightarrow \mathcal{F}_{\alpha}$ and a family $\left(y_{F}\right)_{F \in \mathcal{F}_{\alpha} \backslash\{\emptyset\}}$ in $S_{Z}$ such that for all $F \in \mathcal{F}_{\alpha} \backslash \operatorname{MAX}\left(\mathcal{F}_{\alpha}\right)$ the sequence $\left(y_{F \cup\{n\}}\right)_{n>\max F}$ is a block sequence, and for all $F \in \mathcal{F}_{\alpha}$ we have $\left\|x_{F^{\prime}}-y_{F}\right\|<\varepsilon_{|F|}$. It follows from Proposition 5 that $\alpha<\mathrm{I}_{\mathrm{bl}}\left(\mathcal{F}_{\bar{\varepsilon}}^{E, Z}\right)$.

Proposition 8. Let $Z$ be a Banach space with an FDD $E=\left(E_{i}\right)$. Let $\mathcal{F}$ be a hereditary block tree of $\left(E_{i}\right)$ in $Z$. Then for all $\bar{\varepsilon}=\left(\varepsilon_{i}\right) \subset(0,1)$ and for all limit ordinals $\alpha$, if $\mathrm{I}_{\mathrm{bl}}\left(\mathcal{F}_{\bar{\varepsilon}}^{E, Z}\right)<\alpha$, then $\mathrm{I}_{\mathrm{CB}}(\widetilde{\mathcal{F}})<\alpha$.

The proof consists of two parts. We first replace block sequences of $\left(E_{i}\right)$ with sequences of finite subsets of $\mathbb{N}$ (Lemma 9), and then prove a discrete compression result (Lemma 10). Before we begin we need to extend the notion of block index and related notions to a discrete setting. We write $\Sigma$ for the set of all finite successive sequences in $[\mathbb{N}]^{<\omega} \backslash\{\emptyset\}$ and $S$ for the set of all infinite successive sequences in $[\mathbb{N}]^{<\omega} \backslash\{\emptyset\}$. A tree $\mathcal{G} \subset \Sigma$ on $[\mathbb{N}]^{<\omega}$ will be called a block tree in $[\mathbb{N}]^{<\omega}$, and its $S$-index will be called the block index of $\mathcal{G}$, denoted by $\mathrm{I}_{\mathrm{bl}}(\mathcal{G})$. We shall also use the term "block derivative" and the notation $\mathcal{G}_{\mathrm{bl}}^{\prime}, \mathcal{G}_{\mathrm{bl}}^{(\alpha)}$ just as in the Banach space case.

LEMMA 9. Let $Z$ be a Banach space with an FDD $E=\left(E_{i}\right)$. Let $\mathcal{F}$ be a block tree of $\left(E_{i}\right)$ in $Z$. Let

$$
\begin{aligned}
\operatorname{supp} \mathcal{F}=\left\{\left(A_{i}\right)_{i=1}^{n} \in \Sigma: n \in \mathbb{N}, \exists\left(z_{i}\right)_{i=1}^{n} \in \mathcal{F},\right. & \\
& \left.\operatorname{supp}_{E}\left(z_{i}\right)=A_{i} \text { for } i=1, \ldots, n\right\} .
\end{aligned}
$$

Then for all $\bar{\varepsilon}=\left(\varepsilon_{i}\right) \subset(0,1)$ we have

$$
\mathrm{I}_{\mathrm{bl}}(\operatorname{supp}(\mathcal{F})) \leq \mathrm{I}_{\mathrm{bl}}\left(\mathcal{F}_{\bar{\varepsilon}}^{E, Z}\right) \text {. }
$$

Proof. To simplify notation we are going to write $\mathcal{F}_{\bar{\varepsilon}}$ instead of $\mathcal{F}_{\bar{\varepsilon}}^{E, Z}$. We show by induction that for all $\alpha<\omega_{1}$ we have

$$
(\operatorname{supp}(\mathcal{F}))_{\text {bl }}^{(\alpha)} \subset \operatorname{supp}\left(\left(\mathcal{F}_{\bar{\varepsilon}}\right)_{\mathrm{bl}}^{(\alpha)}\right) \quad \forall \mathcal{F}, \forall \bar{\varepsilon} \text {. }
$$

Lemma 9 will then follow immediately. We begin with the case $\alpha=1$. Let $\left(A_{1}, \ldots, A_{n}\right) \in(\operatorname{supp}(\mathcal{F}))_{\text {bl }}^{\prime}$. Then there is an infinite successive sequence $\left(B_{i}\right)$ in $[\mathbb{N}]^{<\omega} \backslash\{\emptyset\}$ such that $\left(A_{1}, \ldots, A_{n}, B_{i}\right) \in \operatorname{supp}(\mathcal{F})$ for all $i \in \mathbb{N}$. Now choose $\left(z_{1, i}, \ldots, z_{n, i}, z_{i}\right) \in \mathcal{F}$ such that $\operatorname{supp}_{E}\left(z_{k, i}\right)=A_{k}$ for $k=1, \ldots, n$ and $\operatorname{supp}_{E}\left(z_{i}\right)=B_{i}$. By compactness, for some $i_{0} \in \mathbb{N}$ we have $\left(z_{1, i_{0}}, \ldots, z_{n, i_{0}}, z_{i}\right) \in \mathcal{F}_{\bar{\varepsilon}}$ for infinitely many $i \in \mathbb{N}$. It follows that $\left(z_{1, i_{0}}, \ldots, z_{n, i_{0}}\right) \in\left(\mathcal{F}_{\bar{\varepsilon}}\right)_{\mathrm{bl}}^{\prime}$ and $\left(A_{1}, \ldots, A_{n}\right) \in \operatorname{supp}\left(\left(\mathcal{F}_{\bar{\varepsilon}}\right)_{\mathrm{bl}}^{\prime}\right)$, as required. 
In the inductive step we shall use the fact that $\left(\mathcal{F}_{\text {bl }}^{(\alpha)}\right)_{\bar{\varepsilon}} \subset\left(\mathcal{F}_{\bar{\varepsilon}}\right)_{\text {bl }}^{(\alpha)}$ for all $\mathcal{F}, \bar{\varepsilon}, \alpha$, which can be verified by an easy induction. Assume now that (4) holds. We then have

$$
\begin{aligned}
(\operatorname{supp}(\mathcal{F}))_{\mathrm{bl}}^{(\alpha+1)} & =\left((\operatorname{supp}(\mathcal{F}))_{\mathrm{bl}}^{(\alpha)}\right)_{\mathrm{bl}}^{\prime} \\
& \subset\left(\operatorname{supp}\left(\left(\mathcal{F}_{\bar{\varepsilon}}\right)_{\mathrm{bl}}^{(\alpha)}\right)\right)_{\mathrm{bl}}^{\prime} \quad \text { (by the induction hypothesis) } \\
& \subset \operatorname{supp}\left(\left(\mathcal{H}_{\bar{\varepsilon}}\right)_{\mathrm{bl}}^{\prime}\right) \quad\left(\text { by the case } \alpha=1, \text { where } \mathcal{H}=\left(\mathcal{F}_{\bar{\varepsilon}}\right)_{\mathrm{bl}}^{(\alpha)}\right) \\
& \subset \operatorname{supp}\left(\left(\mathcal{F}_{2 \bar{\varepsilon}}\right)_{\mathrm{bl}}^{(\alpha+1)}\right) .
\end{aligned}
$$

This proves (4) with $\alpha$ replaced by $\alpha+1$.

Finally, let $\lambda$ be a limit ordinal and assume that (4) holds for all $\alpha<\lambda$. We have

$$
(\operatorname{supp}(\mathcal{F}))_{\mathrm{bl}}^{(\lambda)}=\bigcap_{\alpha<\lambda}(\operatorname{supp}(\mathcal{F}))_{\mathrm{bl}}^{(\alpha)} \subset \bigcap_{\alpha<\lambda} \operatorname{supp}\left(\left(\mathcal{F}_{\bar{\varepsilon}}\right)_{\mathrm{bl}}^{(\alpha)}\right) \subset \operatorname{supp}\left(\left(\mathcal{F}_{2 \bar{\varepsilon}}\right)_{\mathrm{bl}}^{(\lambda)}\right) .
$$

The first inclusion follows from the induction hypothesis. To see the second inclusion fix a sequence $\left(\alpha_{i}\right)$ of ordinals with $\alpha_{i} \nearrow \lambda$ and assume that

$$
\left(A_{1}, \ldots, A_{n}\right) \in \operatorname{supp}\left(\left(\mathcal{F}_{\bar{\varepsilon}}\right)_{\mathrm{bl}}^{\left(\alpha_{i}\right)}\right) \quad \text { for all } i \in \mathbb{N} .
$$

For each $i \in \mathbb{N}$ choose

$$
\left(z_{1, i}, \ldots, z_{n, i}\right) \in\left(\mathcal{F}_{\bar{\varepsilon}}\right)_{\mathrm{bl}}^{\left(\alpha_{i}\right)}
$$

such that $\operatorname{supp}_{E}\left(z_{k, i}\right)=A_{k}$ for $k=1, \ldots, n$. By compactness, we find $i_{0} \in \mathbb{N}$ such that for infinitely many $i \in \mathbb{N}$ we have

$$
\left\|z_{k, i}-z_{k, i_{0}}\right\|<\varepsilon_{k} \quad \text { for } k=1, \ldots, n .
$$

It follows that $\left(z_{1, i_{0}}, \ldots, z_{n, i_{0}}\right) \in\left(\mathcal{F}_{2 \bar{\varepsilon}}\right)_{\mathrm{bl}}^{\left(\alpha_{i}\right)}$ for infinitely many $i \in \mathbb{N}$, and hence $\left(z_{1, i_{0}}, \ldots, z_{n, i_{0}}\right) \in\left(\mathcal{F}_{2 \bar{\varepsilon}}\right)_{\mathrm{bl}}^{(\lambda)}$. In turn this implies that $\left(A_{1}, \ldots, A_{n}\right) \in$ $\operatorname{supp}\left(\left(\mathcal{F}_{2 \bar{\varepsilon}}\right)_{\text {bl }}^{(\lambda)}\right)$, as required.

Lemma 10. Let $\mathcal{G} \subset \Sigma$ be a hereditary block tree in $[\mathbb{N}]^{<\omega}$, and let $\min \mathcal{G}=\left\{F \in[\mathbb{N}]^{<\omega}: \exists\left(A_{1}, \ldots, A_{|F|}\right) \in \mathcal{G}, F=\left\{\min A_{i}: i=1, \ldots,|F|\right\}\right\}$. Then for any limit ordinal $\alpha$, if $\mathrm{I}_{\mathrm{bl}}(\mathcal{G})<\alpha$, then $\mathrm{I}_{\mathrm{CB}}(\min \mathcal{G})<\alpha$.

Proof. We are going to show the following three statements:

(i) For all $n<\omega$ we have $(\min \mathcal{G})_{\mathrm{CB}}^{(2 n+2)} \subset \min \left(\mathcal{G}_{\mathrm{bl}}^{(n+1)}\right)$.

(ii) Let $\alpha$ be a limit ordinal. If

$$
(\min \mathcal{G})_{\mathrm{CB}}^{(\alpha)} \subset \bigcap_{\beta<\alpha} \min \left(\mathcal{G}_{\mathrm{bl}}^{(\beta)}\right),
$$

then for all $n<\omega$ we have

$$
(\min \mathcal{G})_{\mathrm{CB}}^{(\alpha+2 n+1)} \subset \min \left(\mathcal{G}_{\mathrm{bl}}^{(\alpha+n)}\right) .
$$


(iii) For every limit ordinal $\alpha<\omega_{1}$ we have

$$
(\min \mathcal{G})_{\mathrm{CB}}^{(\alpha)} \subset \bigcap_{\beta<\alpha} \min \left(\mathcal{G}_{\mathrm{bl}}^{(\beta)}\right) .
$$

Since the functions $\mathrm{I}_{\mathrm{CB}}(\cdot)$ and $\mathrm{I}_{\mathrm{bl}}(\cdot)$ only take successor ordinal values, statement (iii) implies the lemma immediately. We start with a proof of (i) and (ii) in the case $n=0$. The general case in both parts follows by an easy induction.

Let $\left(m_{1}, \ldots, m_{k}\right)$ be an element of $(\min \mathcal{G})_{\mathrm{CB}}^{\prime \prime}$. Then there exist $m \in \mathbb{N}$ and an infinite subset $N$ of $\mathbb{N}$ with $m_{k}<m<\min N$ such that

$$
\left(m_{1}, \ldots, m_{k}, m, n\right) \in \min \mathcal{G} \quad \text { for all } n \in N \text {. }
$$

For each $n \in N$ choose $\left(A_{1, n}, \ldots, A_{k, n}, B_{n}, C_{n}\right) \in \mathcal{G}$ such that

$$
\left(\min A_{1, n}, \ldots, \min A_{k, n}, \min B_{n}, \min C_{n}\right)=\left(m_{1}, \ldots, m_{k}, m, n\right) .
$$

After passing to a subsequence we can assume that $A_{j, n}=A_{j}$ for $j=1, \ldots, k$ and for all $n \in N$. Since $\mathcal{G}$ is hereditary, we have $\left(A_{1}, \ldots, A_{k}, C_{n}\right) \in \mathcal{G}$ for all $n \in N$, and hence $\left(A_{1}, \ldots, A_{k}\right) \in \mathcal{G}_{\mathrm{bl}}^{\prime}$. It follows that $\left(m_{1}, \ldots, m_{k}\right) \in$ $\min \left(\mathcal{G}_{\mathrm{bl}}^{\prime}\right)$, which completes the proof of (i), $n=0$.

To show (ii) for $n=0$, fix a sequence $\left(\beta_{i}\right)$ of ordinals with $\beta_{i} \nearrow \alpha$. Pick an element $\left(m_{1}, \ldots, m_{k}\right) \in(\min \mathcal{G})_{\mathrm{CB}}^{(\alpha+1)}$. Then there exist $m \in \mathbb{N}$ with $m_{k}<m$ such that

$$
\left(m_{1}, \ldots, m_{k}, m\right) \in(\min \mathcal{G})_{\mathrm{CB}}^{(\alpha)} \subset \min \left(\mathcal{G}_{\mathrm{bl}}^{\left(\beta_{i}\right)}\right) \quad \text { for all } i \in \mathbb{N} .
$$

For each $i \in \mathbb{N}$ choose $\left(A_{1, i}, \ldots, A_{k, i}, B_{i}\right) \in \mathcal{G}_{\mathrm{bl}}^{\left(\beta_{i}\right)}$ such that

$$
\left(\min A_{1, i}, \ldots, \min A_{k, i}, \min B_{i}\right)=\left(m_{1}, \ldots, m_{k}, m\right) \text {. }
$$

After passing to a subsequence we can assume that $A_{j, i}=A_{j}$ for $j=$ $1, \ldots, k$ and for all $i \in \mathbb{N}$. Since $\left(A_{1}, \ldots, A_{k}\right) \in \mathcal{G}_{\mathrm{bl}}^{\left(\beta_{i}\right)}$ for all $i \in \mathbb{N}$, we have $\left(A_{1}, \ldots, A_{k}\right) \in \mathcal{G}_{\mathrm{bl}}^{(\alpha)}$ and $\left(m_{1}, \ldots, m_{k}\right) \in \min \left(\mathcal{G}_{\mathrm{bl}}^{(\alpha)}\right)$, as required.

Finally, we are going to show (iii) by induction on $\alpha$. It follows from (i) that (5) holds for $\alpha=\omega$. Moreover, if (5) holds for a limit ordinal $\alpha$, then it also holds for $\alpha+\omega$ by (ii). Now, assume that $\alpha$ is the limit of a strictly increasing sequence $\left(\alpha_{n}\right)$ of non-zero limit ordinals and that (iii) holds with $\alpha$ replaced by $\alpha_{n}$ for all $n \in \mathbb{N}$. Then, in particular, we have

$$
(\min \mathcal{G})_{\mathrm{CB}}^{\left(\alpha_{n+1}\right)} \subset \min \left(\mathcal{G}_{\mathrm{bl}}^{\left(\alpha_{n}\right)}\right) \quad \text { for all } n \in \mathbb{N},
$$

from which (iii) follows immediately for $\alpha$.

5. The Szlenk index. Here we recall the definition and basic properties of the Szlenk index. We then recall or prove further properties that are relevant for our purposes. A fairly comprehensive survey on the Szlenk index can be found in [17]. 
Let $X$ be a separable Banach space, and let $K$ be a non-empty, $w^{*}$ compact subset of $X^{*}$. For $\varepsilon>0$ set

$$
K_{\varepsilon}^{\prime}=\left\{x^{*} \in K: \forall w^{*} \text {-neighbourhoods } U \text { of } x^{*}, \operatorname{diam}(K \cap U)>\varepsilon\right\},
$$

where $\operatorname{diam}(K \cap U)$ denotes the norm-diameter of $K \cap U$. We now define $K_{\varepsilon}^{(\alpha)}$ for each countable ordinal $\alpha$ by recursion as follows:

$$
\begin{aligned}
K_{\varepsilon}^{(0)} & =K, & & \\
K_{\varepsilon}^{(\alpha+1)} & =\left(K_{\varepsilon}^{(\alpha)}\right)_{\varepsilon}^{\prime} & & \text { for all } \alpha<\omega_{1}, \\
K_{\varepsilon}^{(\lambda)} & =\bigcap_{\alpha<\lambda} K_{\varepsilon}^{(\alpha)} & & \text { for a limit ordinal } \lambda<\omega_{1} .
\end{aligned}
$$

Next, we associate to $K$ the following ordinal indices:

$$
\eta(K, \varepsilon)=\sup \left\{\alpha<\omega_{1}: K_{\varepsilon}^{(\alpha)} \neq \emptyset\right\} \quad \text { and } \quad \eta(K)=\sup _{\varepsilon>0} \eta(K, \varepsilon) .
$$

Finally, we define the Szlenk index $\operatorname{Sz}(X)$ of $X$ to be $\eta\left(B_{X^{*}}\right)$, where $B_{X^{*}}$ is the unit ball of $X^{*}$.

REMARK. The original definition in [26] is slightly different, but it gives the same ordinal index.

Szlenk used his index to show that there is no separable, reflexive space universal for the class of all separable, reflexive spaces. This result follows immediately from the following properties of the function $\mathrm{Sz}(\cdot)$.

Theorem 11 ([26]). Let $X$ and $Y$ be separable Banach spaces.

(i) $X^{*}$ is separable if and only if $\mathrm{Sz}(X)<\omega_{1}$.

(ii) If $X$ isomorphically embeds into $Y$, then $\mathrm{Sz}(X) \leq \mathrm{Sz}(Y)$.

(iii) For all $\alpha<\omega_{1}$ there exists a separable, reflexive space with Szlenk index at least $\alpha$.

We next restate in one theorem a number of results from [1] in our terminology. This includes an expression of the Szlenk index in terms of the weak index of certain trees.

TheOREM 12 ([1]). Let $X$ be a separable, infinite-dimensional Banach space not containing $\ell_{1}$. For $\varrho \in(0,1)$ let

$$
\mathcal{F}_{\varrho}=\left\{\left(x_{i}\right)_{i=1}^{n} \in S_{X}^{<\omega}: n \in \mathbb{N},\left\|\sum_{i=1}^{n} a_{i} x_{i}\right\| \geq \varrho \sum_{i=1}^{n} a_{i} \forall\left(a_{i}\right)_{i=1}^{n} \subset \mathbb{R}^{+}\right\} .
$$

Then

$$
\mathrm{Sz}(X)=\sup _{\varrho>0} \mathrm{I}_{\mathrm{W}}\left(\mathcal{F}_{\varrho}\right) .
$$

Moreover, if $X^{*}$ is separable, then for some $\alpha<\omega_{1}$, we have $\operatorname{Sz}(X)=\omega^{\alpha}$ and the above supremum is not attained. 
We next consider the Szlenk index of sums of Banach spaces. For finite sums (Proposition 14) this can be computed directly from the definition using a kind of "Leibniz" rule for higher order derivatives of products of $w^{*}$-compact sets (Lemma 13(iii)). For infinite sums (Proposition 15) we use the weak index as well as the result on finite sums to obtain an upper bound. In what comes we will denote by $\alpha \boxplus \beta$ the "pointwise sum" of ordinals $\alpha$ and $\beta$, which is defined as follows. We first write $\alpha$ and $\beta$ in Cantor Normal Form as

$$
\alpha=\omega^{\gamma_{1}} \cdot m_{1}+\omega^{\gamma_{2}} \cdot m_{2}+\cdots+\omega^{\gamma_{k}} \cdot m_{k}
$$

and

$$
\beta=\omega^{\gamma_{1}} \cdot n_{1}+\omega^{\gamma_{2}} \cdot n_{2}+\cdots+\omega^{\gamma_{k}} \cdot n_{k},
$$

where $k \in \mathbb{N}, \gamma_{1}>\cdots>\gamma_{k} \geq 0$ are ordinals, and $m_{i}, n_{i}<\omega$ for all $i$. Then we set

$$
\alpha \boxplus \beta=\omega^{\gamma_{1}} \cdot\left(m_{1}+n_{1}\right)+\omega^{\gamma_{2}} \cdot\left(m_{2}+n_{2}\right)+\cdots+\omega^{\gamma_{k}} \cdot\left(m_{k}+n_{k}\right) .
$$

Lemma 13. Let $X$ and $Y$ be separable Banach spaces. Let $m \in \mathbb{N}$, let $K, K_{1}, \ldots, K_{m}$ be non-empty, $w^{*}$-compact subsets of $X^{*}$, and let $L$ be a non-empty, $w^{*}$-compact subset of $Y^{*}$. Let $\varepsilon>0$.

(i) $\left(\bigcup_{j=1}^{m} K_{j}\right)_{\varepsilon}^{\prime} \subset \bigcup_{j=1}^{m}\left(K_{j}\right)_{\varepsilon / 2}^{\prime}$.

(ii) For the subset $K \times L$ of $X^{*} \oplus_{\infty} Y^{*}=\left(X \oplus_{1} Y\right)^{*}$ we have

$$
(K \times L)_{\varepsilon}^{\prime}=K_{\varepsilon}^{\prime} \times L \cup K \times L_{\varepsilon}^{\prime} .
$$

(iii) For the subset $K \times L$ of $X^{*} \oplus_{\infty} Y^{*}$ and for any ordinal $\alpha<\omega_{1}$ we have

$$
(K \times L)_{\varepsilon}^{(\alpha)} \subset \bigcup_{\beta \boxplus \gamma=\alpha} K_{\varepsilon / 2}^{(\beta)} \times L_{\varepsilon / 2}^{(\gamma)}
$$

Proof. (i) Let $x^{*} \in\left(\bigcup_{j=1}^{m} K_{j}\right)_{\varepsilon}^{\prime}$. Since the $w^{*}$-topology on a $w^{*}$-compact subset of $X^{*}$ is metrizable, it follows easily from the definition that there is a sequence $\left(x_{n}^{*}\right)$ in $\bigcup_{j=1}^{m} K_{j}$ such that $x_{n}^{*} \stackrel{w^{*}}{\rightarrow} x^{*}$ as $n \rightarrow \infty$, and $\left\|x_{n}^{*}-x^{*}\right\|>\varepsilon / 2$ for all $n \in \mathbb{N}$. After passing to a subsequence we may assume that for some $1 \leq j \leq m$ we have $x_{n}^{*} \in K_{j}$, and hence $x^{*} \in\left(K_{j}\right)_{\varepsilon / 2}^{\prime}$.

(ii) This is immediate from the definition and from the fact that we are working with the $\ell_{\infty}$-sum of $X^{*}$ and $Y^{*}$.

(iii) We prove this statement by induction. The case $\alpha=0$ is clear. Using parts (i) and (ii) and assuming the statement for some $\alpha$, we 
have

$$
\begin{aligned}
(K \times L)_{\varepsilon}^{(\alpha+1)} & \subset \bigcup_{\beta \boxplus \gamma=\alpha}\left(K_{\varepsilon / 2}^{(\beta)} \times L_{\varepsilon / 2}^{(\gamma)}\right)_{\varepsilon / 2}^{\prime} \\
& =\bigcup_{\beta \boxplus \gamma=\alpha}\left(K_{\varepsilon / 2}^{(\beta+1)} \times L_{\varepsilon / 2}^{(\gamma)} \cup K_{\varepsilon / 2}^{(\beta)} \times L_{\varepsilon / 2}^{(\gamma+1)}\right) \\
& =\bigcup_{\beta \boxplus \gamma=\alpha+1} K_{\varepsilon / 2}^{(\beta)} \times L_{\varepsilon / 2}^{(\gamma)} .
\end{aligned}
$$

Finally, if $\lambda$ is a limit ordinal, then by the induction hypothesis we have

$$
(K \times L)_{\varepsilon}^{(\lambda)} \subset \bigcap_{\alpha<\lambda}\left(\bigcup_{\beta \boxplus \gamma=\alpha} K_{\varepsilon / 2}^{(\beta)} \times L_{\varepsilon / 2}^{(\gamma)}\right) .
$$

Write $\lambda$ in Cantor Normal Form:

$$
\lambda=\omega^{\lambda_{1}} \cdot m_{1}+\cdots+\omega^{\lambda_{k}} \cdot m_{k}
$$

with $m_{k}>0$, and for all $n \in \mathbb{N}$ set

$$
\alpha_{n}=\omega^{\lambda_{1}} \cdot m_{1}+\cdots+\omega^{\lambda_{k}} \cdot\left(m_{k}-1\right)+\omega^{\delta_{n}} \cdot n,
$$

where $\lambda_{k}=\delta_{n}+1$ for all $n \in \mathbb{N}$ if $\lambda_{k}$ is a successor ordinal, and $\delta_{n} \nearrow \lambda_{k}$ if $\lambda_{k}$ is a limit ordinal.

Now let $x^{*}=\left(y^{*}, z^{*}\right) \in(K \times L)_{\varepsilon}^{(\lambda)}$. For each $n \in \mathbb{N}$ we have $\alpha_{n}<\lambda$, so there exist ordinals $\beta_{n}$ and $\gamma_{n}$ with $\beta_{n} \boxplus \gamma_{n}=\alpha_{n}$ and $x^{*} \in K_{\varepsilon / 2}^{\left(\beta_{n}\right)} \times L_{\varepsilon / 2}^{\left(\gamma_{n}\right)}$. Now there exist $n_{1}, \ldots, n_{k}, p_{1}, \ldots, p_{k}<\omega$ and an infinite subset $N$ of $\mathbb{N}$ such that

$$
\begin{aligned}
& \beta_{n}=\omega^{\lambda_{1}} \cdot n_{1}+\cdots+\omega^{\lambda_{k}} \cdot n_{k}+\omega^{\delta_{n}} \cdot u_{n}, \\
& \gamma_{n}=\omega^{\lambda_{1}} \cdot p_{1}+\cdots+\omega^{\lambda_{k}} \cdot p_{k}+\omega^{\delta_{n}} \cdot v_{n},
\end{aligned}
$$

where $u_{n}+v_{n}=n$ for all $n \in N$. Assume $\sup _{n} u_{n}=\omega\left(\right.$ the case $\sup _{n} v_{n}=\omega$ being similar). Set

$$
\beta=\omega^{\lambda_{1}} \cdot n_{1}+\cdots+\omega^{\lambda_{k}} \cdot\left(n_{k}+1\right), \quad \gamma=\omega^{\lambda_{1}} \cdot p_{1}+\cdots+\omega^{\lambda_{k}} \cdot p_{k} .
$$

Then $\beta=\sup _{n} \beta_{n}$, whereas $\gamma \leq \gamma_{n}$ for all $n \in N$. It follows that $y^{*} \in K_{\varepsilon / 2}^{(\beta)}=$ $\bigcap_{n} K_{\varepsilon / 2}^{\left(\beta_{n}\right)}$, and $z^{*} \in L_{\varepsilon / 2}^{(\gamma)}$. Since $\beta \boxplus \gamma=\lambda$, statement (iii) with $\lambda$ replacing $\alpha$ follows.

Proposition 14. Let $X$ and $Y$ be separable Banach spaces. Then

$$
\mathrm{Sz}\left(X \oplus_{1} Y\right)=\max \{\mathrm{Sz}(X), \mathrm{Sz}(Y)\} \text {. }
$$

Proof. The inequality $\mathrm{Sz}\left(X \oplus_{1} Y\right) \geq \max \{\mathrm{Sz}(X), \mathrm{Sz}(Y)\}$ follows immediately from Theorem 11(ii). The reverse inequality is trivial if either both $X$ and $Y$ are finite-dimensional, or one of $X$ and $Y$ has non-separable dual. So we can assume by the last part of Theorem 12 that $\max \{\operatorname{Sz}(X), \operatorname{Sz}(Y)\}=\omega^{\eta}$ for some $0<\eta<\omega_{1}$. 
Now let $K=B_{X^{*}}, L=B_{Y^{*}}$ and set $\alpha=\omega^{\eta} \cdot 2+1$. Applying part (iii) of Lemma 13 we obtain

$$
\left(B_{\left(X \oplus_{1} Y\right)^{*}}\right)_{\varepsilon}^{(\alpha)}=\left(B_{X^{*}} \times B_{Y^{*}}\right)_{\varepsilon}^{(\alpha)}=\emptyset .
$$

It follows that $\mathrm{Sz}\left(X \oplus_{1} Y\right)<\alpha$, and hence $\operatorname{Sz}\left(X \oplus_{1} Y\right) \leq \omega^{\eta}$, as required.

Proposition 15. Let $\left(X_{n}\right)$ be a sequence of separable Banach spaces. Let $X=\left(\bigoplus_{n} X_{n}\right)_{\ell_{2}}$ be the $\ell_{2}$-sum of $\left(X_{n}\right)$, and let $\alpha$ be a countable ordinal. If $\mathrm{Sz}\left(X_{n}\right) \leq \omega^{\alpha}$ for all $n \in \mathbb{N}$, then $\mathrm{Sz}(X) \leq \omega^{\alpha+1}$.

In the proof we shall use the following notation. For $n \in \mathbb{N}$ we denote by $P_{n}$ the canonical projection of $X$ onto $X_{n}$, i.e., for $x=\left(x_{i}\right) \in X$ with $x_{i} \in X_{i}$ for all $i \in \mathbb{N}$ we have $P_{n}(x)=x_{n}$. For a finite subset $A$ of $\mathbb{N}$ we let $P_{A}=\sum_{n \in A} P_{n}$.

Proof. Assume for a contradiction that $\mathrm{Sz}(X)>\omega^{\alpha+1}$. By Theorem 12 there exists $\varrho \in(0,1)$ such that setting

$$
\mathcal{F}=\left\{\left(x_{i}\right)_{i=1}^{n} \in S_{X}^{<\omega}: n \in \mathbb{N},\left\|\sum a_{i} x_{i}\right\| \geq \varrho \sum a_{i} \forall\left(a_{i}\right)_{i=1}^{n} \subset \mathbb{R}^{+}\right\}
$$

we have $\mathrm{I}_{\mathrm{w}}(\mathcal{F})>\omega^{\alpha+1}$. Note that by the geometric form of the Hahn-Banach theorem, $\left(x_{1}, \ldots, x_{n}\right) \in S_{X}^{<\omega}$ belongs to $\mathcal{F}$ if and only if there exists $x^{*} \in S_{X^{*}}$ such that $x^{*}\left(x_{i}\right) \geq \varrho$ for each $i=1, \ldots, n$.

We are going to show the following claim. Let $\mathbf{x}=\left(x_{1}, \ldots, x_{m}\right) \in S_{X}^{<\omega}$, and let $k \geq 1, M \geq 0$ be integers. Assume that $\mathrm{I}_{\mathrm{w}}(\mathcal{F}(\mathbf{x}))>\omega^{\alpha} \cdot k$. Then there exists $\mathbf{y}=\left(y_{1}, \ldots, y_{n}\right) \in S_{X}^{<\omega}$ such that

(i) $\mathrm{I}_{\mathrm{w}}(\mathcal{F}(\mathbf{x}, \mathbf{y}))>\omega^{\alpha} \cdot(k-1)$,

(ii) for all $x^{*} \in S_{X^{*}}$ there exists $i \in\{1, \ldots, n\}$, with $x^{*}\left(P_{[1, M]}\left(y_{i}\right)\right)<\varrho / 4$.

Let us first see how this claim completes the proof. Fix $K \in \mathbb{N}$ with $K>4 / \varrho^{2}$. We obtain sequences $\mathbf{x}_{1}, \ldots, \mathbf{x}_{K}$ in $S_{X}^{<\omega}$ and $N_{1}<\cdots<N_{K}$ in $\mathbb{N}$ recursively as follows: at the $j$ th step we apply the claim with $\mathbf{x}=\left(\mathbf{x}_{1}, \ldots, \mathbf{x}_{j-1}\right)$, $k=K-j+1$ and $M=N_{j-1}$ to obtain $\mathbf{y}$ as above (for $j=1$ we begin with $\mathbf{x}=\emptyset, k=K$ and $\left.M=N_{0}=0\right)$. Then we set $\mathbf{x}_{j}=\mathbf{y}$ and choose $N_{j}>N_{j-1}$ such that writing $\mathbf{x}_{j}=\left(y_{j, 1}, \ldots, y_{j, L_{j}}\right)$ we have

$$
\left\|y_{j, l}-P_{\left[1, N_{j}\right]}\left(y_{j, l}\right)\right\|<\varrho / 4 \quad \text { for } l=1, \ldots, L_{j} .
$$

From property (i) we deduce in particular that $\left(\mathbf{x}_{1}, \ldots, \mathbf{x}_{K}\right) \in \mathcal{F}$. Thus there exists $x^{*} \in S_{X^{*}}$ such that

$$
x^{*}\left(y_{j, l}\right) \geq \varrho \quad \text { for all } j=1, \ldots, K, l=1, \ldots, L_{j} .
$$

It follows that for each $j=1, \ldots, K$ we can find $1 \leq l_{j} \leq L_{j}$ such that

$$
x^{*}\left(P_{\left[N_{j-1}+1, N_{j}\right]}\left(y_{j, l_{j}}\right)\right)>\varrho / 2,
$$

and hence we get $\left\|x^{*}\right\| \geq \sqrt{K} \varrho / 2>1$, which is a contradiction. 
We now turn to the proof of the claim. Define

$$
\begin{aligned}
\mathcal{G}=\left\{\left(y_{1}, \ldots, y_{n}\right) \in \mathcal{F}(\mathbf{x}): n \in\right. & \mathbb{N}, \exists x^{*} \in S_{X^{*}}, \\
& \left.x^{*}\left(P_{[1, M]}\left(y_{i}\right)\right) \geq \varrho / 4 \text { for } i=1, \ldots, n\right\} .
\end{aligned}
$$

Note that $\mathcal{G}$ is a tree on $S_{X}^{<\omega}$. If $\mathrm{I}_{\mathrm{w}}(\mathcal{G})>\omega^{\alpha}$, then by Theorem 12 we have $\operatorname{Sz}\left(X_{1} \oplus_{2} \cdots \oplus_{2} X_{M}\right)>\omega^{\alpha}$, contradicting Proposition 14. So we must have $\mathrm{I}_{\mathrm{w}}(\mathcal{G}) \leq \omega^{\alpha}$. On the other hand,

$$
\mathrm{I}_{\mathrm{W}}\left((\mathcal{F}(\mathbf{x}))_{\mathrm{w}}^{\left(\omega^{\alpha} \cdot(k-1)\right)}\right)>\omega^{\alpha} .
$$

Thus we can find

$$
\mathbf{y} \in(\mathcal{F}(\mathbf{x}))_{\mathrm{w}}^{\left(\omega^{\alpha} \cdot(k-1)\right)} \backslash \mathcal{G} .
$$

Properties (i) and (ii) are now easily checked.

Proposition 16. Let $\alpha$ be an ordinal with $1 \leq \alpha<\omega_{1}$. The Szlenk index of the Tsirelson space of order $\alpha$ is given by

$$
\operatorname{Sz}\left(T_{\alpha}\right)=\omega^{\alpha \cdot \omega} .
$$

Proof. For each $\varrho \in(0,1)$ let

$$
\mathcal{F}_{\varrho}=\left\{\left(x_{i}\right)_{i=1}^{n} \in S_{T_{\alpha}}^{<\omega}: n \in \mathbb{N},\left\|\sum_{i=1}^{n} a_{i} x_{i}\right\| \geq \varrho \sum_{i=1}^{n} a_{i} \forall\left(a_{i}\right)_{i=1}^{n} \subset \mathbb{R}^{+}\right\} .
$$

We first show that $\operatorname{Sz}\left(T_{\alpha}\right) \geq \omega^{\alpha \cdot \omega}$. Let $\left(e_{i}\right)$ be the unit vector basis of $T_{\alpha}$. It follows from the definition of $T_{\alpha}$ that for each $n \in \mathbb{N}$, if $F \in \mathcal{S}_{\alpha \cdot n}$, then $\left(e_{i}\right)_{i \in F}$ is $2^{n}$-equivalent to the unit vector basis of $\ell_{1}^{|F|}$, so in particular $\left(e_{i}\right)_{i \in F} \in$ $\mathcal{F}_{2^{-n}}$. Hence by Proposition 5 and Theorem 12 we have

$$
\omega^{\alpha \cdot n}<\mathrm{I}_{\mathrm{W}}\left(\mathcal{F}_{2^{-n}}\right)<\mathrm{Sz}\left(T_{\alpha}\right) .
$$

Since $n \in \mathbb{N}$ was arbitrary, the inequality $\operatorname{Sz}\left(T_{\alpha}\right) \geq \omega^{\alpha \cdot \omega}$ follows at once.

For the reverse inequality assume that $\omega^{\gamma}<\operatorname{Sz}\left(T_{\alpha}\right)$, where $\gamma=\alpha \cdot \omega$. Then by Theorem 12 there exists $\varrho \in(0,1)$ with $\omega^{\gamma}<\mathrm{I}_{\mathrm{w}}\left(\mathcal{F}_{\varrho}\right)$, and by Proposition 5 there is a family $\mathcal{G}=\left(x_{F}\right)_{F \in \mathcal{S}_{\gamma} \backslash\{\emptyset\}} \subset \mathcal{F}_{\varrho}$ such that for all $F \in \mathcal{S}_{\gamma} \backslash \operatorname{MAX}\left(\mathcal{S}_{\gamma}\right)$ the sequence $\left(x_{F \cup\{n\}}\right)_{n>\max F}$ is weakly null.

By standard perturbation arguments we may, after making $\varrho$ smaller and replacing $\left(x_{F}\right)_{F \in \mathcal{S}_{\gamma} \backslash\{\emptyset\}}$ by $\left(x_{F^{\prime}}\right)_{F \in \mathcal{S}_{\gamma} \backslash\{\emptyset\}}$ for an appropriate pruning function $F \mapsto F^{\prime}: \mathcal{S}_{\gamma} \rightarrow \mathcal{S}_{\gamma}$ if necessary, assume that $\mathcal{G}$ is a block tree of $\left(e_{i}\right)$ in $T_{\alpha}$.

We now apply a result of $\mathrm{R}$. Judd and the first named author [16]. In their terminology $\mathcal{G}$ is an $\ell_{1}-K$-block basis tree of $\left(e_{i}\right)$ in $T_{\alpha}$ with $K=\varrho^{-1}$ (we are using the 1-unconditionality of $\left.\left(e_{i}\right)\right)$, and hence its order $\mathrm{o}(\mathcal{G})$ is at most the Bourgain $\ell_{1}$-index of $T_{\alpha}$, which is shown in [16] to be $\omega^{\gamma}$. On the other hand, by Proposition 5 we have $\mathrm{o}(\mathcal{G})>\omega^{\gamma}$. This contradiction completes the proof. 
Proposition 17. Let $\alpha$ be a countable ordinal and let $Z$ be a Banach space with an $F D D E=\left(E_{i}\right)$ that satisfies subsequential $T_{\alpha}$-upper estimates. Then $\operatorname{Sz}(Z) \leq \omega^{\alpha \cdot \omega}$.

Proof. For each $\varrho \in(0,1)$ let

$$
\begin{aligned}
& \mathcal{F}_{\varrho}=\left\{\left(x_{i}\right)_{i=1}^{n} \in S_{Z}^{<\omega}: n \in \mathbb{N},\left\|\sum_{i=1}^{n} a_{i} x_{i}\right\| \geq \varrho \sum_{i=1}^{n} a_{i} \forall\left(a_{i}\right)_{i=1}^{n} \subset \mathbb{R}^{+}\right\} . \\
& \mathcal{G}_{\varrho}=\left\{\left(x_{i}\right)_{i=1}^{n} \in S_{T_{\alpha}}^{<\omega}: n \in \mathbb{N},\left\|\sum_{i=1}^{n} a_{i} x_{i}\right\| \geq \varrho \sum_{i=1}^{n} a_{i} \forall\left(a_{i}\right)_{i=1}^{n} \subset \mathbb{R}^{+}\right\} .
\end{aligned}
$$

Fix $\gamma<\operatorname{Sz}(Z)$. By Theorem 12 there exists $\varrho \in(0,1)$ such that $\mathrm{I}_{\mathrm{w}}\left(\mathcal{F}_{\varrho}\right)>\gamma$, and by Proposition 5 there is a family $\left(x_{F}\right)_{F \in \mathcal{F}_{\gamma} \backslash\{\emptyset\}} \subset \mathcal{F}_{\varrho}$ such that for all $F \in \mathcal{F}_{\gamma} \backslash \operatorname{MAX}\left(\mathcal{F}_{\gamma}\right)$ the sequence $\left(x_{F \cup\{n\}}\right)_{n>\max F}$ is weakly null.

By standard perturbation arguments we may, after making $\varrho$ smaller and appropriately pruning $\left(x_{F}\right)_{F \in \mathcal{F}_{\gamma} \backslash\{\emptyset\}}$ if necessary, assume that $\left(x_{F}\right)_{F \in \mathcal{F}_{\gamma} \backslash\{\emptyset\}}$ is a block tree of $\left(E_{i}\right)$ in $Z$, and that for all $F \in \mathcal{F}_{\gamma} \backslash \operatorname{MAX}\left(\mathcal{F}_{\gamma}\right)$ the sequence $\left(x_{F \cup\{n\}}\right)_{n>\max F}$ is a block basis of $\left(E_{i}\right)$. Let $\left(e_{i}\right)$ be the unit vector basis of $T_{\alpha}$ and define

$$
t_{F}=e_{\operatorname{minsupp}_{E}\left(x_{F}\right)} \quad \text { for all } F \in \mathcal{F}_{\gamma} \backslash\{\emptyset\} .
$$

Note that $\left(t_{F}\right)_{F \in \mathcal{F}_{\gamma} \backslash\{\emptyset\}}$ is a block tree of $\left(e_{i}\right)$ in $T_{\alpha}$ and that it is contained in $\mathcal{G}_{\varrho^{\prime}}$ for some $\varrho^{\prime} \in(0,1)$ since $\left(E_{i}\right)$ satisfies subsequential $T_{\alpha^{-}}$upper estimates. Since $\left(e_{i}\right)$ is shrinking, it follows by Proposition 5 that $\gamma<\mathrm{I}_{\mathrm{w}}\left(\mathcal{G}_{\varrho^{\prime}}\right)$. Using Theorem 12 and Proposition 16 we deduce that $\mathrm{Sz}(Z) \leq \omega^{\alpha \cdot \omega}$, as required.

REMARK. It follows from properties of higher order Tsirelson spaces (Proposition 3) that the unit vector basis of $T_{\alpha}^{*}$ satisfies subsequential $T_{\alpha^{-}}$ upper estimates. Hence the above result shows that

$T_{\alpha} \in \mathcal{C}_{\omega^{\alpha \cdot \omega}}=\left\{X: X\right.$ is separable, reflexive, $\left.\max \left\{\operatorname{Sz}(X), \operatorname{Sz}\left(X^{*}\right)\right\} \leq \omega^{\alpha \cdot \omega}\right\}$.

\section{The main theorem and its consequences}

THEOREM 18. Let $Z$ be a Banach space with a shrinking, bimonotone $F D D\left(E_{i}\right)$ and let $X$ be an infinite-dimensional closed subspace of $Z$. Then for any $C>4$ there exist an ordinal $\alpha<\mathrm{Sz}(X)$, a sequence $\bar{\delta}=\left(\delta_{i}\right) \subset(0,1)$ with $\delta_{i} \downarrow 0$, and a blocking $\left(G_{i}\right)$ of $\left(E_{i}\right)$ with $G_{i}=\bigoplus_{j=m_{i-1}}^{m_{i}-1} E_{j}, i \in \mathbb{N}$, $1=m_{0}<m_{1}<m_{2}<\cdots$, such that if $\left(x_{i}\right) \subset S_{X}$ is a $\bar{\delta}$-block sequence of $\left(G_{n}\right)$ with $\left\|x_{i}-P_{\left(s_{i-1}, s_{i}\right]}^{G} x_{i}\right\|<\delta_{i}$ for all $i \in \mathbb{N}, 1 \leq s_{0}<s_{1}<s_{2}<\cdots$, then $\left(x_{i}\right)$ is $C$-dominated by $\left(e_{m_{s_{i-1}}}\right)$, where $\left(e_{i}\right)$ is the unit vector basis of $T_{\mathcal{F}_{\alpha}, 1 / 2}$.

We first prove some consequences of Theorem 18. In Corollary 20 below we recast the property of being in the class $\mathcal{C}_{\alpha}$ in terms of certain lower and 
upper Tsirelson-norm estimates. In Theorem 21 we show that for certain values of $\alpha$ these estimates are best possible, which proves Theorem A from the Introduction. These norm estimates and Theorem 1 are the two main ingredients in answering Pełczyński's question, which we do in Theorem 22 followed by a refinement in Theorem 23. We then state a result of Johnson which we use to deduce basis versions (Theorems B and C) of Theorem 23. The rest of the section is taken up by the proof of Theorem 18 .

Corollary 19. Let $X$ be an infinite-dimensional Banach space with separable dual. There exists an ordinal $\alpha<\mathrm{Sz}(X)$ such that $X$ satisfies subsequential $C-T_{\mathcal{F}_{\gamma}, 1 / 2}$-upper tree estimates for any ordinal $\gamma \geq \alpha$, where $C$ is a universal constant.

Proof. By Zippin's theorem [28], $X$ K-embeds into a Banach space $Z$ with a shrinking, bimonotone FDD $\left(E_{i}\right)$, where $K$ is a universal constant. Renorming $X$ with a $K$-equivalent norm we may assume without loss of generality that $X$ is a subspace of $Z$. We now apply Theorem 18 to obtain $\alpha<\operatorname{Sz}(X)$, a sequence $\bar{\delta}=\left(\delta_{i}\right) \subset(0,1), \delta_{i} \downarrow 0$, and a blocking $\left(G_{i}\right)$ of $\left(E_{i}\right)$ with $G_{i}=\bigoplus_{j=m_{i-1}}^{m_{i}-1} E_{j}, i \in \mathbb{N}, 1=m_{0}<m_{1}<\cdots$, such that if $\left(x_{i}\right) \subset S_{X}$ is a $\bar{\delta}$-block sequence of $\left(G_{n}\right)$ with $\left\|x_{i}-P_{\left(s_{i-1}, s_{i}\right]}^{G} x_{i}\right\|<\delta_{i}$ for all $i \in \mathbb{N}$, $1 \leq s_{0}<s_{1}<\cdots$, then $\left(x_{i}\right)$ is 5 -dominated by $\left(e_{\alpha, m_{s_{i-1}}}\right)$, where $\left(e_{\alpha, i}\right)$ is the unit vector basis of $T_{\mathcal{F}_{\alpha}, 1 / 2}$.

Fix an ordinal $\gamma \geq \alpha$ and an integer $l$ such that $\left(e_{\alpha, i}\right)_{i \geq l}$ is 1-dominated by $\left(e_{\gamma, i}\right)_{i \geq l}$ (such an integer exists by property (1) of the fine Schreier families). We now show that $X$ satisfies subsequential $C-T_{\mathcal{F}_{\gamma}, 1 / 2}$-upper tree estimates with $C=5$. Let $\left(x_{t}\right)_{t \in T_{\infty}^{\text {even }}}$ be a normalized, weakly null even tree in $X$. We will inductively choose sequences $s_{0}<s_{1}<\cdots$ and $n_{1}<n_{2}<\cdots$ in $\mathbb{N}$ as follows. Set $s_{0}=1$ and $n_{1}=\max \left(l, m_{1}\right)$. Assume that for some $i \in \mathbb{N}$ we have already chosen $s_{0}<s_{1}<\cdots<s_{i-1}$ and $n_{1}<n_{2}<\cdots<n_{2 i-1}$. Since nodes are weakly null, there exists $n_{2 i}>n_{2 i-1}$ such that

$$
\left\|P_{\left[1, s_{i-1}\right]}^{G} x_{\left(n_{1}, \ldots, n_{2 i}\right)}\right\|<\delta_{i} .
$$

Then choose $s_{i}>s_{i-1}$ such that

$$
\left\|x_{\left(n_{1}, \ldots, n_{2 i}\right)}-P_{\left(s_{i-1}, s_{i}\right]}^{G} x_{\left(n_{1}, \ldots, n_{2 i}\right)}\right\|<\delta_{i} .
$$

Finally, choose $n_{2 i+1}>n_{2 i}$ with $n_{2 i+1} \geq m_{s_{i}}$. This completes the recursive construction. It follows immediately from the choice of $\alpha, \bar{\delta},\left(G_{i}\right)$ and $l$, and from the 1-right-dominant property of $\left(e_{\alpha, i}\right)$, that $\left(x_{\left(n_{1}, \ldots, n_{2 i}\right)}\right)$ is 5 -dominated by $\left(e_{\gamma, n_{2 i-1}}\right)$.

Corollary 20. Let $X$ be an infinite-dimensional, separable, reflexive Banach space. Then there exists an ordinal $\gamma<\max \left\{\operatorname{Sz}(X), \operatorname{Sz}\left(X^{*}\right)\right\}$ such that $X$ satisfies subsequential $C-\left(T_{\mathcal{F}_{\delta}, 1 / 2}^{*}, T_{\mathcal{F}_{\delta}, 1 / 2}\right)$ tree estimates for any $\delta \geq \gamma$, where $C$ is a universal constant. 
Proof. By Corollary 19 there is a universal constant $C$, and there exist ordinals $\alpha<\mathrm{Sz}(X)$ and $\beta<\mathrm{Sz}\left(X^{*}\right)$, such that $X$ satisfies subsequential $C-T_{\mathcal{F}_{\gamma}, 1 / 2^{-}}$upper tree estimates for any $\gamma \geq \alpha$, and $X^{*}$ satisfies subsequential $C-T_{\mathcal{F}_{\delta}, 1 / 2}$-upper tree estimates for any $\delta \geq \beta$. It follows from Proposition 2 that $X$ satisfies subsequential $(2 C+\varepsilon)-\left(T_{\mathcal{F}_{\delta}, 1 / 2}^{*}, T_{\mathcal{F}_{\delta}, 1 / 2}\right)$ tree estimates for any $\varepsilon>0$ and for any $\delta \geq \max \{\alpha, \beta\}$.

The above results show that higher order Tsirelson spaces are more than just mere examples in the hierarchy $\left(\mathcal{C}_{\alpha}\right)_{\alpha<\omega_{1}}$. Indeed they are intimately related to the Szlenk index of an arbitrary separable, reflexive space and its dual. The next theorem shows that this relationship is tight in the classes $\mathcal{C}_{\omega^{\alpha} \cdot \omega}$ : Tsirelson spaces of order $\alpha$ and their duals are maximal and, respectively, minimal in these classes. In particular, this proves Theorem A stated in the Introduction. The proof uses some further results from [22] which we shall not state here as Theorem 21 will not be used in the proof of our universality results.

TheOREm 21. Let $\alpha<\omega_{1}$. For a separable, reflexive space $X$ the following are equivalent.

(i) $X \in \mathcal{C}_{\omega^{\alpha \cdot \omega}}$.

(ii) $X$ satisfies subsequential $\left(T_{\alpha, c}^{*}, T_{\alpha, c}\right)$ tree estimates for some $c \in$ $(0,1)$.

(iii) $X$ embeds into a separable, reflexive space $Z$ with an $F D D\left(E_{i}\right)$ which satisfies subsequential $\left(T_{\alpha, c}^{*}, T_{\alpha, c}\right)$ estimates in $Z$ for some $c \in(0,1)$.

Proof. "(i) $\Rightarrow$ (ii)" By Corollary 20 there exists $n<\omega$ such that $X$ satisfies subsequential $\left(T_{\alpha \cdot n}^{*}, T_{\alpha \cdot n}\right)$ tree estimates. It is not hard to show directly from the definition that the norms $\|\cdot\|_{T_{\alpha \cdot n}}$ and $\|\cdot\|_{T_{\alpha, c}}$ on $c_{00}$, where $c=1 / 2^{1 / n}$, are equivalent. Hence (ii) follows.

"(ii) $\Rightarrow$ (iii)" This is immediate from [22, Theorem 15]. We note that the implication "(iii) $\Rightarrow$ (ii)" is straightforward from the definition.

"(iii) $\Rightarrow(\mathrm{i})$ " Let $Z$ be the space given by (iii), and choose $n \in \mathbb{N}$ such that $c^{n} \leq 1 / 2$. It follows directly from the definition that the unit vector basis of $T_{\alpha, c}$ is dominated by the unit vector basis of $T_{\alpha \cdot n, c^{n}}$, which in turn is dominated by the unit vector basis of $T_{\alpha \cdot n}$. Hence by Theorem 11(ii), and by Propositions 17 and 16, we have

$$
\mathrm{Sz}(X) \leq \mathrm{Sz}(Z) \leq \mathrm{Sz}\left(T_{\alpha \cdot n}\right)=\omega^{\alpha \cdot n \cdot \omega}=\omega^{\alpha \cdot \omega} .
$$

(Alternatively, one can just observe that the proof of Proposition 16 works for $T_{\alpha, c}$, i.e., we have $\operatorname{Sz}\left(T_{\alpha, c}\right)=\omega^{\alpha \cdot \omega}$.) Now since $X$ satisfies (ii), it follows from duality (Proposition 2 and [22, Corollary 14]) that (ii), and hence (iii), also hold with $X$ replaced by $X^{*}$. This gives $\operatorname{Sz}\left(X^{*}\right) \leq \omega^{\alpha \cdot \omega}$. Thus $X \in \mathcal{C}_{\omega^{\alpha \cdot \omega}}$, as required. 
REMARK. Using the proof of Proposition 16 one can show that $\mathrm{Sz}\left(T_{\mathcal{F}_{\alpha}, c}\right)$ $=\alpha^{\omega}$ whenever $1 \leq \alpha<\omega_{1}$ and $c \in(0,1)$. Considering the Cantor Normal Form of $\alpha$, it is possible to write $\alpha^{\omega}=\omega^{\beta \cdot \omega}$ for some $\beta \leq \alpha$. Thus, it is not possible to obtain a finer gradation of the hierarchy $\left(\mathcal{C}_{\alpha}\right)_{\alpha<\omega_{1}}$ by using fine Schreier families.

We are now in a position to answer Pełczyński's question. We shall use the notation

$$
\mathcal{A}_{\alpha}(C)=\mathcal{A}_{T_{\alpha}^{*}, T_{\alpha}}(C) \text { and } \mathcal{A}_{\alpha}=\bigcup_{C<\infty} \mathcal{A}_{\alpha}(C),
$$

where $0<\alpha<\omega_{1}$ and $C \in[1, \infty$ ) (see also the notation preceding Theorem 1). Recall that

$$
\mathcal{C}_{\alpha}=\left\{X: X \text { is separable, reflexive, } \max \left\{\operatorname{Sz}(X), \operatorname{Sz}\left(X^{*}\right)\right\} \leq \alpha\right\},
$$

and that the Szlenk index of an infinite-dimensional Banach space with separable dual is of the form $\omega^{\eta}$ for some $0<\eta<\omega_{1}$ (Theorem 12), so we need only consider the classes $\mathcal{C}_{\alpha}$ when $\alpha$ is of this form. We should also comment on finite-dimensional spaces before proceeding.

For $\alpha<\omega$ we have $\mathcal{C}_{\alpha}=\mathcal{C}_{0}$ is the class of all finite-dimensional spaces. Let $Z$ be the $\ell_{2}$-sum of a countable, dense (with respect to the Banach-Mazur distance) subset of $\mathcal{C}_{0}$. Then by Proposition 15 we have $Z \in \mathcal{C}_{\omega}$. Moreover, $Z$ is universal for $\mathcal{C}_{0}$ : for all $X \in \mathcal{C}_{0}$ and for all $\varepsilon>0, X(1+\varepsilon)$-embeds into $Z$.

For $\omega \leq \alpha<\omega_{1}$ we have $\ell_{2} \in C_{\alpha}$, and hence $\ell_{2} \oplus X \in \mathcal{C}_{\alpha}$ for any finitedimensional space $X$. Thus we can restrict attention to infinite-dimensional spaces for the purpose of finding a universal space for the class $\mathcal{C}_{\alpha}$.

TheOREM 22. For every ordinal $\alpha$ with $0<\alpha<\omega_{1}$ there is a separable, reflexive space with an FDD which is universal for the class $\mathcal{C}_{\omega^{\alpha}}$. More precisely, there is a universal constant $K$ such that for all $0<\alpha<\omega_{1}$ there exists a space $Z \in \mathcal{C}_{\omega^{\alpha} \cdot \omega}$ with an $F D D$ such that every space $X \in \mathcal{C}_{\omega^{\alpha}}$ $K$-embeds into $Z$.

Proof. Let $C \in[1, \infty)$ be the universal constant of Corollary 20, and let $B, D \in[1, \infty)$ be the universal constants of Proposition 3. Let $K=$ $K_{B, D, 1,1}(C)$ be the constant from Theorem 1. Given $0<\alpha<\omega_{1}$, let $Z \in \mathcal{A}_{\alpha}$ be the universal space given by Theorem 1 with $U=T_{\alpha}$ and $V=U^{*}$. In particular $Z$ has an FDD $\left(E_{i}\right)$ that satisfies subsequential $\left(T_{\alpha}^{*}, T_{\alpha}\right)$ estimates in $Z$. By an easy duality argument the $\operatorname{FDD}\left(E_{i}^{*}\right)$ of $Z^{*}$ satisfies subsequential $\left(T_{\alpha}^{*}, T_{\alpha}\right)$ estimates in $Z^{*}$. Hence by Proposition 17 we have $\max \left\{\operatorname{Sz}(Z), \operatorname{Sz}\left(Z^{*}\right)\right\} \leq \omega^{\alpha \cdot \omega}$, i.e., $Z \in \mathcal{C}_{\omega^{\alpha \cdot \omega}}$.

Now let $X \in \mathcal{C}_{\omega^{\alpha}}$ be an infinite-dimensional space. By Corollary 20 we have $X \in \mathcal{A}_{\alpha}(C)$, and hence $X K$-embeds into $Z$. 
Remark. By a result of Johnson and Odell [15], the space $Z$ constructed in the proof of Theorem 22 cannot be in the class $\mathcal{C}_{\omega^{\alpha}}$. Indeed, if that was the case, then every space that embeds into $Z$ would in fact $K$-embed into $Z$. Such a space is called elastic in [15], where it is proved that a separable, elastic space contains $\mathrm{c}_{0}$. Obviously, $Z$ cannot contain $\mathrm{c}_{0}$, giving the required contradiction.

Note that the above theorem yields a universal space for the class $\mathcal{C}_{\omega^{\alpha \cdot \omega}}$ that lives in the class $\mathcal{C}_{\omega^{\alpha \cdot \omega^{2}}}$. A small modification of the proof gives the slightly better result mentioned in the Introduction:

THEOREM 23. For every $\alpha<\omega_{1}$ there is a space $Z_{\alpha} \in \mathcal{C}_{\omega^{\alpha \cdot \omega+1}}$ with an FDD which is universal for the class $\mathcal{C}_{\omega^{\alpha} \cdot \omega}$. More precisely, there is a universal constant $K$, and for each $\alpha<\omega_{1}$ there is a sequence $\left(Z_{\alpha, n}\right)_{n=1}^{\infty}$ of spaces with FDDs in $\mathcal{C}_{\omega^{\alpha \cdot \omega}}$ such that for all $X \in \mathcal{C}_{\omega^{\alpha \cdot \omega}}$ there exists $n \in \mathbb{N}$ such that $X K$-embeds into $Z_{\alpha, n}$. The space $Z_{\alpha}$ can then be taken to be the $\ell_{2}$-direct sum of the sequence $\left(Z_{\alpha, n}\right)_{n=1}^{\infty}$.

Proof. For $\alpha=0$ we have already done this just before stating Theorem 22. Now assume that $0<\alpha<\omega_{1}$, and let $C, K$ be the constants defined in the proof of Theorem 22. Let $Z_{\alpha, n} \in \mathcal{A}_{\alpha \cdot n}$ be the universal space given by Theorem 1 with $U=T_{\alpha \cdot n}$ and $V=U^{*}$. As in the proof of Theorem 22 we deduce that $\max \left\{\mathrm{Sz}\left(Z_{\alpha, n}\right), \operatorname{Sz}\left(Z_{\alpha, n}^{*}\right)\right\} \leq \omega^{\alpha \cdot n \cdot \omega}=\omega^{\alpha \cdot \omega}$, i.e., $Z_{\alpha, n} \in \mathcal{C}_{\omega^{\alpha \cdot \omega}}$. Now let $Z_{\alpha}=\left(\bigoplus_{n=1}^{\infty} Z_{\alpha, n}\right)_{\ell_{2}}$ be the $\ell_{2}$-direct sum of the sequence $\left(Z_{\alpha, n}\right)_{n=1}^{\infty}$. By Proposition 15 we have $Z_{\alpha} \in \mathcal{C}_{\omega^{\alpha \cdot \omega+1}}$.

Finally, let $X \in \mathcal{C}_{\omega^{\alpha \cdot \omega}}$ be an infinite-dimensional space. By Corollary 20 there exists $n \in \mathbb{N}$ such that $X \in \mathcal{A}_{\alpha \cdot n}(C)$, and hence $X K$-embeds into $Z_{\alpha, n}$ and into $Z_{\alpha}$.

As indicated in the Introduction, Theorems B and C now follow from Theorem 23 by applying the following result of Johnson [14].

Theorem 24 ([14, Theorem A]). Let $\left(G_{i}\right)$ be a sequence of finite-dimensional Banach spaces so that

(i) if $E$ is a finite-dimensional Banach space and $\varepsilon>0$, then there is an $i \in \mathbb{N}$ so that $d\left(E, G_{i}\right)=\inf \left\{\|T\| \cdot\left\|T^{-1}\right\|: T: E \rightarrow G_{i}\right.$ is an isomorphism $\}<1+\varepsilon$,

(ii) for each $i \in \mathbb{N}$ there is an infinite $J \subset \mathbb{N}$ so that $G_{i}$ and $G_{j}$ are isometric for all $j \in J$.

Let $C_{2}=\left(\bigoplus_{i=1}^{\infty} G_{i}\right)_{\ell_{2}}$ and let $X$ be any separable space which has the $\lambda$ metric approximation property for some $\lambda \geq 1$. Then $X \oplus C_{2}$ has a basis.

Note that the $\lambda$-metric approximation property is also known as the $\lambda$-bounded approximation property. 
Proof of Theorems $B$ and $C$. Clearly, spaces $X$ with an FDD have the $\lambda$-metric approximation property for some $\lambda \geq 1$, meaning that for any compact set $K \subset X$ and $\varepsilon>0$ there is a finite rank operator $T$ with $\|T(x)-x\|<\varepsilon$ for all $x \in K$. Let $C_{2}$ be the space defined in Theorem 24, and let $Z_{\alpha}$ and $Z_{\alpha, n}, n \in \mathbb{N}$, be the spaces from Theorem 23. Then $Z_{\alpha} \oplus C_{2}$ and $Z_{\alpha, n} \oplus C_{2}$ have Schauder bases and it follows from Propositions 14 and 15 that $\mathrm{Sz}\left(Z_{\alpha} \oplus C_{2}\right)=\mathrm{Sz}\left(Z_{\alpha}\right)=\omega^{\alpha \cdot \omega+1}$ and $\mathrm{Sz}\left(Z_{\alpha, n} \oplus C_{2}\right)=\omega^{\alpha \cdot \omega}$.

In the remainder of this section we give a proof of our main result, Theorem 18, which is at the heart of our embedding and universality results.

Proof of Theorem 18. Fix a constant $D$ with $4<D<C$, and choose $\varrho \in(0,1)$ such that $4+12 \varrho D<D$. Set

$$
\mathcal{F}=\left\{\left(x_{i}\right) \in S_{X}^{<\omega}:\left\|\sum a_{i} x_{i}\right\| \geq 2 \varrho \sum a_{i} \text { for all }\left(a_{i}\right) \subset[0, \infty)\right\} .
$$

Note that $\mathcal{F}$ is a hereditary tree on $S_{X}^{<\omega}$. Next fix a sequence $\bar{\varepsilon}=\left(\varepsilon_{i}\right) \subset$ $(0,1 / 2)$ such that

$$
\mathcal{F}_{10 \bar{\varepsilon}}^{Z} \subset\left\{\left(z_{i}\right) \in S_{Z}^{<\omega}:\left\|\sum a_{i} z_{i}\right\| \geq \varrho \sum a_{i} \text { for all }\left(a_{i}\right) \subset \mathbb{R}^{+}\right\} .
$$

Now consider the hereditary block tree $\mathcal{G}=\Sigma(E, Z) \cap \mathcal{F}_{\bar{\varepsilon}}^{Z}$ of $\left(E_{i}\right)$ in $Z$ and its compression $\widetilde{\mathcal{G}}$. Let $\alpha$ be the Cantor-Bendixson index of $\widetilde{\mathcal{G}}$. By Proposition 6 and by Theorem 12 we have

$$
\mathrm{I}_{\mathrm{w}}\left(\mathcal{F}_{2 \bar{\varepsilon}}^{Z}\right) \leq \mathrm{I}_{\mathrm{w}}\left(\mathcal{F}_{10 \bar{\varepsilon}}^{X}\right)<\mathrm{Sz}(X) .
$$

Since $\mathcal{G}_{\bar{\varepsilon}}^{E, Z} \subset \mathcal{F}_{2 \bar{\varepsilon}}^{Z}$, we have $\mathrm{I}_{\mathrm{bl}}\left(\mathcal{G}_{\bar{\varepsilon}}^{E, Z}\right) \leq \mathrm{I}_{\mathrm{w}}\left(\mathcal{F}_{2 \bar{\varepsilon}}^{Z}\right)$. Since $\mathrm{Sz}(X)$ is a limit ordinal, it follows by Proposition 8 that

$$
\alpha=\mathrm{I}_{\mathrm{CB}}(\widetilde{\mathcal{G}})<\mathrm{Sz}(X) .
$$

We now apply Theorem 4 to obtain an infinite subset $M=\left\{m_{1}, m_{2}, \ldots\right\}$ of $\mathbb{N}$ such that

$$
\operatorname{MAX}\left(\mathcal{F}_{\alpha}\right) \cap[M]^{<\omega} \cap \widetilde{\mathcal{G}}=\emptyset .
$$

To see this, give each element $A$ of the thin family $\operatorname{MAX}\left(\mathcal{F}_{\alpha}\right)$ colour red if $A \in \widetilde{\mathcal{G}}$, and colour blue otherwise, and obtain $M=\left\{m_{1}, m_{2}, \ldots\right\} \in[\mathbb{N}]^{\omega}$ such that $\operatorname{MAX}\left(\mathcal{F}_{\alpha}\right) \cap[M]^{<\omega}$ is monochromatic. Now the map $i \mapsto m_{i}: \mathbb{N} \rightarrow M$ induces a homeomorphism $[\mathbb{N}]^{<\omega} \rightarrow[M]^{<\omega}$ that maps $\mathcal{F}_{\alpha}$ onto $\mathcal{F}_{\alpha} \cap[M]^{<\omega}$ (as $\mathcal{F}_{\alpha}$ is a spreading). Since the Cantor-Bendixson index is a topological invariant, it follows that $\mathrm{I}_{\mathrm{CB}}\left(\mathcal{F}_{\alpha} \cap[M]^{<\omega}\right)=\alpha+1$. Hence $\operatorname{MAX}\left(\mathcal{F}_{\alpha}\right) \cap[M]^{<\omega}$ cannot be monochromatic red, and thus (6) follows. Observe that if $F \in$ $\widetilde{\mathcal{G}} \cap[M]^{<\omega}$, then $F \in \mathcal{F}_{\alpha}$.

Without loss of generality we may assume that $m_{1}>1$. We set $m_{0}=1$ and $G_{i}=\bigoplus_{j=m_{i-1}}^{m_{i}-1} E_{j}$ for all $i \in \mathbb{N}$. Finally, we choose $\bar{\delta}=\left(\delta_{i}\right) \subset(0,1), \delta_{i} \downarrow 0$, 
such that

$$
4 \sum_{i=1}^{\infty} \delta_{i}<\min (\varrho, C-D) \quad \text { and } \quad 4 \sum_{j \geq i} \delta_{j}<\varepsilon_{i} \quad \text { for all } i \in \mathbb{N} .
$$

We will now show that for these choices of $\alpha, \bar{\delta}$ and $\left(G_{i}\right)$ the conclusion of the theorem holds.

Let $\left(x_{i}\right) \subset S_{X}$ be a $\bar{\delta}$-block sequence of $\left(G_{n}\right)$ with $\left\|x_{i}-P_{\left(s_{i-1}, s_{i}\right]}^{G} x_{i}\right\|<\delta_{i}$ for all $i \in \mathbb{N}$ and $1 \leq s_{0}<s_{1}<s_{2}<\cdots$. Set

$$
z_{i}=\frac{P_{\left(s_{i-1}, s_{i}\right]}^{G} x_{i}}{\| P_{\left(s_{i-1}, s_{i}\right]}^{x_{i} \|}} \quad \text { for all } i \in \mathbb{N} \text {. }
$$

Note that $\left\|x_{i}-z_{i}\right\|<2 \delta_{i}$ for all $i \in \mathbb{N}$. Replacing each $z_{i}$ by a small perturbation of itself, if necessary, we can assume that $\min _{\operatorname{supp}}\left(z_{i}\right)=s_{i-1}+1$ and $\min \operatorname{supp}_{E}\left(z_{i}\right)=m_{s_{i-1}}$ for all $i \in \mathbb{N}$. We are going to show that for any $\left(a_{i}\right) \in \mathrm{c}_{00}$ we have

$$
\left\|\sum a_{i} z_{i}\right\| \leq D\left\|\sum a_{i} e_{m_{s_{i-1}}}\right\|
$$

It then follows easily from the choice of $\bar{\delta}$ that $\left(x_{i}\right)$ is $C$-dominated by $\left(e_{m_{s_{i-1}}}\right)$. The proof of (7) proceeds by induction on the size of the support of $\left(a_{i}\right)$. If this is 1 , then the statement is clear. In general, we begin by choosing $z^{*} \in B_{Z^{*}}$ such that

$$
\left\|\sum a_{i} z_{i}\right\|=\sum a_{i} z^{*}\left(z_{i}\right)
$$

We then consider the set

$$
I=\left\{i \in \mathbb{N}:\left|z^{*}\left(z_{i}\right)\right| \geq 3 \varrho\right\},
$$

which splits into $I^{+}=\left\{i \in \mathbb{N}: z^{*}\left(z_{i}\right) \geq 3 \varrho\right\}$ and $I^{-}=I \backslash I^{+}$. For a finite set $F \subset \mathbb{N}$ we shall write $\operatorname{ms}(F)$ for the set $\left\{m_{s_{i-1}}: i \in F\right\}$. We claim that $\operatorname{ms}\left(I^{+}\right)$and $\operatorname{ms}\left(I^{-}\right)$belong to $\mathcal{F}_{\alpha}$. Indeed, by the choice of $\bar{\delta}$, for any $\left(b_{i}\right)_{i \in I^{+}} \subset \mathbb{R}^{+}$we have

$$
\left\|\sum_{i \in I^{+}} b_{i} x_{i}\right\| \geq \sum_{i \in I^{+}} b_{i} z^{*}\left(z_{i}\right)-\sum_{i \in I^{+}} b_{i} \cdot 2 \delta_{i} \geq 2 \varrho \sum_{i \in I^{+}} b_{i} .
$$

This shows that $\left(x_{i}\right)_{i \in I^{+}}$belongs to $\mathcal{F}$. It follows that $\left(z_{i}\right)_{i \in I^{+}} \in \mathcal{G}$, and $\operatorname{ms}\left(I^{+}\right) \in \widetilde{\mathcal{G}} \cap[M]^{<\omega} \subset \mathcal{F}_{\alpha}$, as required. A similar argument, using $-z^{*}$ instead of $z^{*}$, shows that $\operatorname{ms}\left(I^{-}\right) \in \mathcal{F}_{\alpha}$.

We next partition $\operatorname{supp}\left(a_{i}\right) \backslash I$ into sets $J_{1}<\cdots<J_{l}$, where $l \in \mathbb{N}$ and

$$
\begin{gathered}
3 \varrho<\left\|\left.z^{*}\right|_{\operatorname{span}\left\{z_{i}: i \in J_{k}\right\}}\right\| \leq 6 \varrho \quad \text { for } 1 \leq k<l, \\
\left\|\left.z^{*}\right|_{\operatorname{span}\left\{z_{i}: i \in J_{l}\right\}}\right\| \leq 6 \varrho .
\end{gathered}
$$

This is clearly possible by the definition of $I$ and by the bimonotonicity of $\left(E_{i}\right)$. Set $F=\left\{\min J_{k}: k=1, \ldots, l-1\right\}$. We claim that $\operatorname{ms}(F) \in \mathcal{F}_{\alpha} \backslash$ 
$\operatorname{MAX}\left(\mathcal{F}_{\alpha}\right)$, from which it follows that $\operatorname{ms}(\widetilde{F}) \in \mathcal{F}_{\alpha}$, where $\widetilde{F}=F \cup\left\{\min J_{l}\right\}$. To prove the claim first choose for each $k=1, \ldots, l-1$ a vector $u_{k}=$ $\sum_{i \in J_{k}} c_{i} z_{i} \in S_{Z}$ such that $\sum c_{i} z^{*}\left(z_{i}\right)>3 \varrho$. We can assume without loss of generality that $c_{\min J_{k}} \neq 0$, i.e., that $\min \operatorname{supp}_{E}\left(u_{k}\right)=m_{s_{\min J_{k}-1}}$. Set $\widetilde{v}_{k}=\sum_{i \in J_{k}} c_{i} x_{i}$ and $v_{k}=\widetilde{v}_{k} /\left\|\widetilde{v}_{k}\right\|$ for each $k=1, \ldots, l-1$, and note that

$$
\left\|v_{k}-u_{k}\right\| \leq 2\left\|\widetilde{v}_{k}-u_{k}\right\| \leq 2 \sum_{i \in J_{k}}\left|c_{i}\right| \cdot 2 \delta_{i} \leq 4 \sum_{i \geq k} \delta_{i} .
$$

It follows that for any $\left(b_{k}\right)_{k=1}^{l-1} \subset \mathbb{R}^{+}$we have

$$
\left\|\sum_{k=1}^{l-1} b_{k} v_{k}\right\| \geq \sum_{k=1}^{l-1} b_{k} z^{*}\left(u_{k}\right)-\sum_{k=1}^{l-1} b_{k} \cdot\left\|v_{k}-u_{k}\right\| \geq 2 \varrho \sum_{k=1}^{l-1} b_{k} .
$$

We deduce that $\left(v_{k}\right) \in \mathcal{F},\left(u_{k}\right) \in \mathcal{G}$ and $\operatorname{ms}(F) \in \widetilde{\mathcal{G}} \cap[M]^{<\omega} \subset \mathcal{F}_{\alpha} \backslash \operatorname{MAX}\left(\mathcal{F}_{\alpha}\right)$, as claimed.

The following sequence of inequalities now completes the proof of (7):

$$
\begin{aligned}
\left\|\sum a_{i} z_{i}\right\|= & \sum a_{i} z^{*}\left(z_{i}\right) \leq \sum_{i \in I^{+}}\left|a_{i}\right|+\sum_{i \in I^{-}}\left|a_{i}\right|+\sum_{k=1}^{l} 6 \varrho \cdot\left\|\sum_{i \in J_{k}} a_{i} z_{i}\right\| \\
\leq & 2\left\|\sum_{i \in I^{+}} a_{i} e_{m_{s_{i-1}}}\right\|_{T_{\mathcal{F}_{\alpha}, 1 / 2}}+2\left\|\sum_{i \in I^{-}} a_{i} e_{m_{s_{i-1}}}\right\|_{T_{\mathcal{F}_{\alpha}, 1 / 2}} \\
& +6 \varrho \cdot D \sum_{k=1}^{l}\left\|\sum_{i \in J_{k}} a_{i} e_{m_{s_{i-1}}}\right\|_{T_{\mathcal{F}_{\alpha}, 1 / 2}} \\
\leq & (4+12 \varrho D) \cdot\left\|\sum a_{i} e_{m_{s_{i-1}}}\right\|_{T_{\mathcal{F}_{\alpha}, 1 / 2}} \leq D\left\|\sum a_{i} e_{m_{s_{i-1}}}\right\|_{T_{\mathcal{F}_{\alpha}, 1 / 2}} .
\end{aligned}
$$

It is the third line where we apply the induction hypothesis. Note that by (8) (and since $12 \varrho<1$ ), each $J_{k}$ has size strictly smaller than that of the support of $\left(a_{i}\right)$.

7. Further remarks. In [11] the following universality result is proved.

TheOREM 25 ([11]). For every countable ordinal $\xi$ there is a space $Y_{\xi}$ with separable dual such that every Banach space $X$ with $\mathrm{Sz}(X) \leq \xi$ embeds into $Y_{\xi}$.

This result of P. Dodos and V. Ferenczi is similar to our universality results, but the methods used are completely different. Note that unlike Theorems 22 and 23, the above result does not give information on the Szlenk index of the universal space $Y_{\xi}$. The reason for this is that the use of descriptive set theory in proving results like Theorem 25 yields existence proofs, whereas our approach is more constructive. 
In this final section we describe the setting in which descriptive set theory can be used to study universality problems for certain classes of separable Banach spaces. We shall also explain what is missing if one tries to use this approach to prove the main results of our paper. For an extensive survey on the interplay between descriptive set theory and Banach spaces the reader is referred to the Handbook article by Argyros, Godefroy and Rosenthal [3].

Recall that every separable Banach space is a subspace of $C[0,1]$, the space of continuous functions on the Cantor set. The set SB of all closed subspaces of $C[0,1]$ is given the Effros-Borel structure, which is the $\sigma$-algebra generated by the sets $\{F \in \mathrm{SB}: F \cap U \neq \emptyset\}$, where $U$ ranges over all open subsets of $C[0,1]$. This allows one to study classes of Banach spaces according to their descriptive complexity and apply results of descriptive set theory. This has been first formalized by B. Bossard [6], and then taken up by S. Argyros and P. Dodos [2] to study universality problems. One of the central notions introduced in [2] is the following.

Definition. A class $\mathcal{C}$ of separable Banach space in SB is said to be strongly bounded if for every analytic subset $\mathcal{A}$ of $\mathcal{C}$ there exists $Y \in \mathcal{C}$ that contains isomorphic copies of every $X \in \mathcal{A}$.

The main result of [11] is that the classes $\mathcal{S R}$ of separable, reflexive spaces and $\mathcal{S D}$ of spaces with separable dual are strongly bounded. Since $\{X \in \mathcal{S D}: \mathrm{Sz}(X) \leq \xi\}$ is analytic (even Borel, which was proved in [6]), Theorem 25 follows. However, it was not known whether the classes $\mathcal{C}_{\alpha}$ from Pełczyński's question were analytic or not, and so the main theorem from [11] could not be applied. From our results we can now prove the following.

THEOREM 26. For every countable ordinal $\alpha$ the class $\mathcal{C}_{\alpha}$ is analytic in the Effros-Borel structure of SB.

Proof. Fix a countable ordinal $\alpha$. We begin by showing that the class $\mathcal{C}_{\omega^{\alpha \cdot \omega}}$ is analytic. By Theorem 23, if $X \in \mathcal{C}_{\omega^{\alpha \cdot \omega}}$, then there exists $n \in \mathbb{N}$ such that $X$ isomorphically embeds into $Z_{\alpha, n}$, which we denote by $X \hookrightarrow Z_{\alpha, n}$. Conversely, assume that $X \hookrightarrow Z_{\alpha, n}$. Since $Z_{\alpha, n}$ has an FDD satisfying subsequential $\left(T_{\alpha \cdot n}^{*}, T_{\alpha \cdot n}\right)$ estimates, it follows easily that $X$ satisfies subsequential $\left(T_{\alpha \cdot n}^{*}, T_{\alpha \cdot n}\right)$-tree estimates. By duality the same holds for $X^{*}$, and hence $X^{*}$ also embeds into $Z_{\alpha, n}$. From Proposition 17 we now obtain

$$
\max \left\{\operatorname{Sz}(X), \operatorname{Sz}\left(X^{*}\right)\right\} \leq \operatorname{Sz}\left(Z_{\alpha, n}\right) \leq \omega^{\alpha \cdot n},
$$

and so $X \in \mathcal{C}_{\omega^{\alpha \cdot \omega}}$.

It is well known and easy to show that for any $Y \in$ SB the set $\{X \in \mathrm{SB}$ : $X \hookrightarrow Y\}$ is analytic. It follows that

$$
\mathcal{C}_{\omega^{\alpha \cdot \omega}}=\bigcup_{n \in \mathbb{N}}\left\{X \in \mathrm{SB}: X \hookrightarrow Z_{\alpha, n}\right\}
$$

is analytic, as claimed. 
To prove the general case, we use a recent result of P. Dodos [10] which states that

$$
\mathcal{S}_{\alpha}=\left\{X \in \mathrm{SB}: \max \left\{\mathrm{Sz}(X), \mathrm{Sz}\left(X^{*}\right)\right\} \leq \alpha\right\}
$$

is analytic. Since $\mathcal{C}_{\alpha}=\mathcal{S}_{\alpha} \cap \mathcal{C}_{\omega^{\alpha \cdot \omega}}$, it follows immediately that $\mathcal{C}_{\alpha}$ is also analytic.

Remark. As mentioned in the Introduction, it was C. Rosendal who pointed out to us that the analyticity of $\mathcal{C}_{\omega^{\alpha \cdot \omega}}$ follows from our results. Later P. Dodos informed us that this fact together with his result implies the general case.

\section{References}

[1] D. Alspach, R. Judd, and E. Odell, The Szlenk index and local $l_{1}$-indices, Positivity 9 (2005), 1-44.

[2] S. Argyros and P. Dodos, Genericity and amalgamation of classes of Banach spaces, preprint.

[3] S. A. Argyros, G. Godefroy, and H. P. Rosenthal, Descriptive set theory and Banach spaces, in: Handbook of the Geometry of Banach Spaces, Vol. 2, North-Holland, Amsterdam, 2003, 1007-1069.

[4] S. Banach, Théorie des opérations linéaires, Warszawa, 1932.

[5] B. Bossard, An ordinal version of some applications of the classical interpolation theorem, Fund. Math. 152 (1997), 55-74.

[6] -, A coding of separable Banach spaces. Analytic and coanalytic families of Banach spaces, ibid. 172 (2002), 117-152.

[7] J. Bourgain, On separable Banach spaces, universal for all separable reflexive spaces, Proc. Amer. Math. Soc. 79 (1980), 241-246.

[8] P. G. Casazza, W. B. Johnson, and L. Tzafriri, On Tsirelson's space, Israel J. Math. 47 (1984), 81-98.

[9] P. G. Casazza and Th. J. Shura, Tsirel'son's Space, Lecture Notes in Math. 1363, Springer, Berlin, 1989.

[10] P. Dodos, Definability under duality, preprint.

[11] P. Dodos and V. Ferenczi, Some strongly bounded classes of Banach spaces, Fund. Math. 193 (2007), 171-179.

[12] T. Figiel and W. B. Johnson, A uniformly convex Banach space which contains no $l_{p}$, Compos. Math. 29 (1974), 179-190.

[13] G. Godefroy and N. J. Kalton, Isometric embeddings and universal spaces, Extracta Math., to appear.

[14] W. B. Johnson, Factoring compact operators, Israel J. Math. 9 (1971), 337-345.

[15] W. B. Johnson and E. Odell, The diameter of the isomorphism class of a Banach space, Ann. of Math. (2) 162 (2005), 423-437.

[16] R. Judd and E. Odell, Concerning Bourgain's $l_{1}$-index of a Banach space, Israel J. Math. 108 (1998), 145-171.

[17] G. Lancien, A survey on the Szlenk index and some of its applications, RACSAM Rev. R. Acad. Cienc. Exactas Fís. Nat. Ser. A Mat. 100 (2006), 209-235.

[18] D. H. Leung and W.-K. Tang, The Bourgain $\ell^{1}$-index of mixed Tsirelson space, J. Funct. Anal. 199 (2003), 301-331. 
[19] E. Odell and Th. Schlumprecht, Trees and branches in Banach spaces, Trans. Amer. Math. Soc. 354 (2002), 4085-4108.

[20] - A universal reflexive space for the class of uniformly convex Banach spaces, Math. Ann. 335 (2006), 901-916.

[21] —, - Embedding into Banach spaces with finite dimensional decompositions, RACSAM Rev. R. Acad. Cienc. Exactas Fís. Nat. Ser. A Mat. 100 (2006), 295-323.

[22] E. Odell, Th. Schlumprecht, and A. Zsák, A new infinite game in Banach spaces with applications, in: Banach Spaces and their Applications in Analysis (Proc. Internat. Conf. at Miami University, May 22-27, 2006, in honor of Nigel Kalton's 60th birthday), to appear.

[23] A. Pełczyński, Universal bases, Studia Math. 32 (1969), 247-268.

[24] S. Prus, Finite-dimensional decompositions with p-estimates and universal Banach spaces, Bull. Polish Acad. Sci. Math. 31 (1983), 281-288.

[25] P. Pudlák and V. Rödl, Partition theorems for systems of finite subsets of integers, Discrete Math. 39 (1982), 67-73.

[26] W. Szlenk, The non-existence of a separable reflexive Banach space universal for all separable reflexive Banach spaces, Studia Math. 30 (1968), 53-61.

[27] B. S. Tsirel'son, Not every Banach space contains $\ell_{p}$ or $\mathrm{c}_{0}$, Funktsional. Anal. i Prilozhen. 8 (1974), no. 2, 57-60 (in Russian); English transl.: Funct. Anal. Appl. 8 (1974), 138-141.

[28] M. Zippin, Banach spaces with separable duals, Trans. Amer. Math. Soc. 310 (1988), 371-379.

Department of Mathematics

The University of Texas

1 University Station C1200

Austin, TX 78712, U.S.A.

E-mail: odell@math.utexas.edu

School of Mathematical Sciences

University of Nottingham

University Park

Nottingham NG7 2RD, United Kingdom

E-mail: andras.zsak@maths.nottingham.ac.uk
Department of Mathematics

Texas A\&M University College Station, TX 78712, U.S.A. E-mail: schlump@math.tamu.edu

Received April 3, 2007

Revised version August 9, 2007 